NASA Technical Memorandum 106645

ICOMP-94-11; AIAA-95-0160

\title{
Evaluation of Boundary Conditions for Computational Aeroacoustics
}

R. Hixon and S.-H. Shih

Institute for Computational Mechanics in Propulsion

Lewis Research Center

Cleveland, Ohio

and

R.R. Mankbadi

National Aeronautics and Space Administration

Lewis Research Center

Cleveland, Ohio

Prepared for the

33rd Aerospace Sciences Meeting

sponsored by the American Institute of Aeronautics and Astronautics

Reno, Nevada, January 5-12, 1995

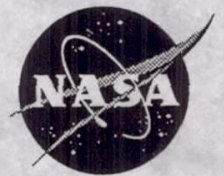

National Aeronautics and Space Administration

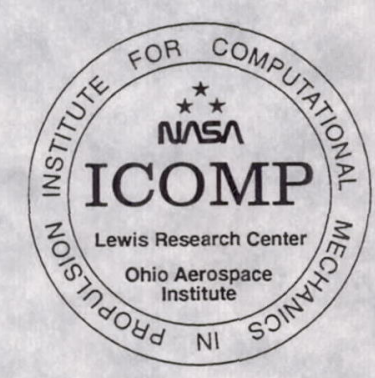




\title{
EVALUATION OF BOUNDARY CONDITIONS FOR COMPUTATIONAL AEROACOUSTICS
}

\author{
R. Hixon* and S.-H. Shih* \\ Institute for Computational Mechanics in Propulsion \\ Lewis Research Center \\ Cleveland, $\mathrm{OH} 44135$ \\ and \\ Reda R. Mankbadi** \\ National Aeronautics and Space Adminsitration \\ Lewis Research Center \\ Cleveland, $\mathrm{OH} 44135$
}

\begin{abstract}
SUMMARY
The performance of three acoustic boundary condition formulations is investigated. The effect of implementation differences is also studied. Details of all implementations are given. Results are shown for the acoustic field of a monopole in a uniform freestream.

\section{INTRODUCTION}

Accurate prediction of flow fluctuations representing sound is the goal of Computational Aeroacoustics (CAA) (see, for instance, Ref. 1). To do this, a grid is constructed which covers the region of interest, and the discretized governing equations are solved on this grid. The computational domain is usually finite, and boundary conditions must be imposed at the edges of the grid. These boundary conditions can generate spurious fluctuations that render the computed solution entirely unacceptable.

Most of the classical boundary treatments used in computational fluid dynamics are concerned only with the steadyflow solution. Several suggestions for boundary conditions for unsteady flows have recently been proposed. These proposals can be classified in three categories: (I) quasi one-dimensional characteristics, (II) decomposition of the solution into Fourier modes, and (III) asymptotic analysis of the governing equations for large distances.

The present work is concerned with evaluating these types of boundary conditions by comparing the numerical results obtained with various boundary conditions to a known analytical solution. The effect of implementation of each boundary condition on its performance is also investigated.
\end{abstract}

\section{GOVERNING EQUATIONS}

The equations to be solved are the Linearized Euler Equations, which may be written in cylindrical coordinates as:

$$
\frac{\partial \tilde{Q}}{\partial \mathrm{t}}+\frac{\partial \tilde{\mathrm{F}}}{\partial \mathrm{x}}+\frac{1}{\mathrm{r}} \frac{\partial(\mathrm{r} \tilde{\mathrm{G}})}{\partial \mathrm{r}}+\frac{1}{\mathrm{r}} \frac{\partial \tilde{\mathrm{H}}}{\partial \theta}=\tilde{\mathrm{S}}
$$

\footnotetext{
${ }^{*}$ Senior Research Associate, ICOMP; Member AIAA.

**Senior Scientist and Leader, Computational Aeroacousticis; Associate Fellow, AIAA.
} 
where:

$$
\begin{gathered}
\tilde{Q}=\left\{\begin{array}{c}
\tilde{\rho} \\
\tilde{u} \\
\tilde{\tilde{w}} \\
\tilde{w} \\
\tilde{e}
\end{array}\right\} \\
\tilde{F}=\left\{\begin{array}{c}
\tilde{p}+2 \tilde{u} U-\tilde{\rho} U^{2} \\
\tilde{u} V+\tilde{v} U-\tilde{\rho} U V \\
\tilde{u} W+\tilde{w} U-\tilde{\rho} U W \\
(\tilde{p}+\tilde{e}) U+(\tilde{u}-\tilde{\rho} U) E
\end{array}\right\} \\
\tilde{G}=\left\{\begin{array}{c} 
\\
\tilde{u} V+\tilde{v} U-\tilde{\rho} U V \\
\tilde{p}+2 \tilde{v} V-\tilde{\rho} V^{2} \\
\tilde{v} W+\tilde{w} V-\tilde{\rho} V W \\
(\tilde{p}+\tilde{e}) V+(\tilde{v}-\tilde{\rho} V) E
\end{array}\right\}, \\
\tilde{H}=\left\{\begin{array}{c} 
\\
\tilde{u} W+\tilde{w} U-\tilde{\rho} U W \\
\tilde{v} W+\tilde{w} V-\tilde{\rho} V W \\
\tilde{p}+2 \tilde{w} W-\tilde{\rho} W W^{2} \\
(\tilde{p}+\tilde{e}) W+(\tilde{w}-\tilde{\rho} W) E
\end{array}\right\},
\end{gathered}
$$

and

$$
\tilde{S}=\left\{\begin{array}{c}
0 \\
0 \\
\tilde{p}+2 \tilde{w} W-\tilde{\rho} W^{2} \\
\tilde{v} W+\tilde{w} V-\tilde{\rho} V W \\
0
\end{array}\right\} .
$$

Here

$$
\begin{gathered}
\tilde{\mathrm{p}}=(\gamma-1),\left[\tilde{\mathrm{e}}-(\tilde{\mathrm{u} U}+\tilde{\mathrm{v}} \mathrm{V}+\tilde{\mathrm{w}} \mathrm{W})+\frac{1}{2} \tilde{\mathrm{\rho}}\left(\mathrm{U}^{2}+\mathrm{V}^{2}+\mathrm{W}^{2}\right)\right] \\
\mathrm{P}=(\gamma-1) \rho\left[\mathrm{E}-\frac{1}{2}\left(\mathrm{U}^{2}+\mathrm{V}^{2}+\mathrm{W}^{2}\right)\right]
\end{gathered}
$$

and

$$
(\tilde{\rho}, \tilde{u}, \tilde{v}, \tilde{w}, \tilde{e})=\left[\rho^{\prime},(\rho u)^{\prime},(\rho v)^{\prime},(\rho w)^{\prime},(\rho e)^{\prime}\right] .
$$

In this notation, $\mathrm{U}$ is the axial mean velocity, $\mathrm{V}$ is the radial mean velocity, and $\mathrm{W}$ is the azimuthal mean velocity. 


\section{NUMERICAL SCHEME}

The code is a modified split MacCormack solver, which is second order accurate in time and fourth order accurate in space. This extension of the MacCormack scheme is known as the 2-4 scheme, and was developed by Gottleib and Turkel. ${ }^{2}$ This scheme has been used successfully before by Farouk et. al. ${ }^{3}$ and Ragab and Sheen ${ }^{4}$ for studying nonlinear instability problems in plane shear layers. Sankar, Reddy, and Hariharan ${ }^{5}$ have evaluated this scheme for aeroacoustics applications. The solution procedure is as follows:

Equation (1) may be rewritten in operator form as:

$$
q^{n+1}=L_{r x} q^{n}
$$

In words, given the flow variables $q$ at a time level ' $n$ ', the code computes derivatives in both the radial and axial directions and uses these quantities to advance the flow field one time step, to the ' $n+1$ ' time level.

In the present code, the operator is split into separate radial and axial contributions:

$$
q^{n+1}=L_{r} L_{x} q^{n}
$$

Each operator solves a portion of Eq. (1):

$$
\begin{gathered}
\mathrm{L}_{\mathrm{r}} \mathrm{q}: \\
\frac{\partial \tilde{Q}}{\partial t}+\frac{1}{\mathrm{r}} \frac{\partial(\mathrm{r} \tilde{\mathrm{G}})}{\partial \mathrm{r}}=\tilde{S} \\
\mathrm{~L}_{\mathrm{x}} \mathrm{q}: \\
\frac{\partial \tilde{\mathrm{Q}}}{\partial \mathrm{t}}+\frac{\partial \tilde{\mathrm{F}}}{\partial \mathrm{x}}=0
\end{gathered}
$$

The operators are applied in a symmetric way to avoid any numerical biasing of the solution:

$$
q^{n+2}=L_{x} L_{r} L_{r} L_{x} q^{n}
$$

Each operator consists of a predictor and a corrector step. Each step uses one-sided differencing:

Predictor:

$$
\begin{gathered}
q^{n+\frac{1}{2}}=q^{n}-\frac{\Delta t}{6 \Delta x}\left(7 F_{i}-8 F_{i-1}+F_{i-2}\right)^{n} \\
q^{n+1}=\frac{1}{2}\left(q^{n}+q^{n+\frac{1}{2}}+\frac{\Delta t}{6 \Delta x}\left(7 F_{i}-8 F_{i+1}+F_{i}+2\right)^{n+\frac{1}{2}}\right)
\end{gathered}
$$

Again, to avoid biasing, the sweep directions are reversed between operators:

$$
\mathrm{q}^{\mathrm{n}+2}=\mathrm{L}_{\mathrm{x}}{ }^{+-} \mathrm{L}_{\mathrm{r}}{ }^{+-} \mathrm{L}_{\mathrm{r}}{ }^{-+} \mathrm{L}_{\mathrm{x}}{ }^{-+} \mathrm{q}^{\mathrm{n}}
$$


At the edges, flux quantities from outside the computational domain are required for the spatial differences. These quantities are computed using third order accurate extrapolations from the interior of the domain:

$$
F_{i+1}=4 F_{i}-6 F_{i-1}+4 F_{i-2}-F_{i-3}
$$

\section{MATHEMATICAL FORMULATION OF BOUNDARY CONDITIONS}

The primary objective in CAA is to accurately compute the oscillatory flow field. Since the computational domain is finite, numerical boundary conditions must be imposed at the grid boundaries. Improper specification of these boundary conditions results in artificial disturbances which can render the computed oscillatory flow solution unacceptable. This problem is most pronounced at subsonic outflows. As such, outflow boundary conditions for subsonic flows have received special attention and will be discussed herein.

\section{THOMPSON (1987)}

Thompson ${ }^{6,7}$ describes how to decompose hyperbolic equations into wave modes of definite velocity and then how to specify boundary conditions for the incoming waves. The starting point of Thompson's analysis is the nonlinear Euler equations. The essence of his approach is that one-dimensional characteristic analysis can be performed by considering the transverse terms as a constant source term. The 1-D characteristic analysis makes clear which waves are propagating into and out of the computational domain. The amplitude of the outward propagating waves are defined entirely from the variables inside the computational domain, while the amplitude of the inward propagating waves are specified as boundary conditions. For nonreflective boundary conditions, the amplitude of the inward propagating waves are set to zero.

To illustrate Thompson's approach, let us consider the nonconservative axisymmetric Euler Equations written in cylindrical coordinates:

$$
Q_{t}+A_{r}+B Q_{x}+C=0
$$

where

$$
\begin{gathered}
Q=\left\{\begin{array}{l}
\rho \\
u \\
v \\
p
\end{array}\right\}, \\
A=\left[\begin{array}{llll}
v & 0 & \rho & 0 \\
0 & v & 0 & 0 \\
0 & 0 & v & \frac{1}{\rho} \\
0 & 0 & c^{2} \rho & v
\end{array}\right], \\
B=\left[\begin{array}{llll}
u & \rho & 0 & 0 \\
0 & u & 0 & \frac{1}{\rho} \\
0 & 0 & u & 0 \\
0 & c^{2} \rho & 0 & u
\end{array}\right],
\end{gathered}
$$


and

$$
C=\frac{1}{r}\left\{\begin{array}{c}
-\rho v \\
0 \\
0 \\
-c^{2} \rho v
\end{array}\right\}
$$

We first consider the radial boundary. The axial derivatives are grouped with the source term to rewrite Eq. (18) as:

$$
\mathrm{Q}_{\mathrm{t}}+\mathrm{AQ}_{\mathrm{r}}+\mathrm{K}=0
$$

Following Thompson's derivation, the matrix A is diagonalized using similarity transformation. The eigen vectors are obtained by solving

$$
\operatorname{det}(\mathrm{A}-\lambda \mathrm{I})=0
$$

where I is the identity matrix. The left and right eigenvectors of A are calculated using

$$
\begin{aligned}
1_{i}{ }^{\mathrm{T}} \mathrm{A} & =\lambda_{\mathrm{i}} \mathrm{l}_{\mathrm{i}}^{\mathrm{T}} \\
\mathrm{Ar} \mathrm{r}_{\mathrm{i}} & =\lambda_{\mathrm{i}} \mathrm{r}_{\mathrm{i}} \\
\mathrm{l}_{\mathrm{i}}{ }^{\mathrm{T}} \mathrm{r}_{\mathrm{j}} & =\delta_{\mathrm{ij}}
\end{aligned}
$$

The matrix $\mathrm{S}$ is formed, where the columns of $\mathrm{S}$ are the right eigenvectors $\mathrm{r}_{\mathrm{i}}$, and the similarity transform is defined:

$$
\Lambda=S^{-1} \mathrm{AS}=\left[\begin{array}{cccc}
\lambda_{1} & 0 & 0 & 0 \\
0 & \lambda_{2} & 0 & 0 \\
0 & 0 & \lambda_{3} & 0 \\
0 & 0 & 0 & \lambda_{4}
\end{array}\right]
$$

Applying this transform to Eq. (23) gives:

$$
S^{-1} Q_{t}+\Lambda S^{-1} Q_{r}+S^{-1} K=0
$$

or

$$
S^{-1} Q_{t}+L+S^{-1} K=0
$$

This leads back to

$$
\mathrm{Q}_{\mathrm{t}}+\mathrm{SL}+\mathrm{K}=0
$$

where:

$$
\mathrm{L}_{\mathrm{i}}=\lambda_{\mathrm{i}} \mathrm{l}_{\mathrm{i}}^{\mathrm{T}} \mathrm{Q}_{\mathrm{r}}
$$


In this case, the eigenvalues are:

$$
\begin{gathered}
\lambda_{1}=v-c \\
\lambda_{2}=v \\
\lambda_{3}=v \\
\lambda_{4}=v+c
\end{gathered}
$$

and the left eigenvectors are:

$$
l_{1}=\left\{\begin{array}{c}
0 \\
0 \\
-\rho c \\
1
\end{array}\right\}, l_{2}=\left\{\begin{array}{c}
c^{2} \\
0 \\
0 \\
-1
\end{array}\right\}, l_{3}=\left\{\begin{array}{l}
0 \\
1 \\
0 \\
0
\end{array}\right\}, 1_{4}=\left\{\begin{array}{c}
0 \\
0 \\
\rho c \\
1
\end{array}\right\}
$$

Eigenvalues 1 and 4 are the velocities of sound waves moving in the negative and positive radial direction; eigenvalue 2 is the velocity for entropy advection; and eigenvalue 3 is the velocity at which vorticity is advected in the radial direction.

Evaluating Eq. (30) gives

$$
\begin{gathered}
\mathrm{L}_{1}=(v-c)\left(\mathrm{p}_{\mathrm{r}}-\rho c \mathrm{v}_{\mathrm{r}}\right) \\
\mathrm{L}_{2}=(\mathrm{v})\left(\mathrm{c}^{2} \rho_{\mathrm{r}}-\mathrm{p}_{\mathrm{r}}\right) \\
\mathrm{L}_{3}=(\mathrm{v})\left(\mathrm{u}_{\mathrm{r}}\right) \\
\mathrm{L}_{4}=(\mathrm{v}+\mathrm{c})\left(\mathrm{p}_{\mathrm{r}}+\rho c \mathrm{v}_{\mathrm{r}}\right)
\end{gathered}
$$

Eq. (28) may be written as:

Solving (34) for the time derivatives, we

$$
\begin{aligned}
c^{2} \rho_{t}-p_{t} & =-L_{2}-c^{2} K_{1}+K_{4}=R_{2} \\
u_{t} & =-L_{3}-K_{2}=R_{3} \\
p_{t}+\rho c v_{t} & =-L_{4}-K_{4}-\rho c K_{3}=R_{4}
\end{aligned}
$$

obtain

$$
\begin{gathered}
\rho_{\mathrm{t}}=\frac{1}{\mathrm{c}^{2}}\left(\mathrm{R}_{2}+\frac{1}{2}\left(\mathrm{R}_{1}+\mathrm{R}_{4}\right)\right) \\
\mathrm{u}_{\mathrm{t}}=\mathrm{R}_{3} \\
\mathrm{v}_{\mathrm{t}}=\frac{1}{2 \rho c}\left(\mathrm{R}_{4}-\mathrm{R}_{1}\right) \\
\mathrm{p}_{\mathrm{t}}=\frac{1}{2}\left(\mathrm{R}_{1}+\mathrm{R}_{4}\right)
\end{gathered}
$$

To apply the nonreflective Thompson boundary conditions, it is necessary to determine which waves $\left(L_{1}-L_{4}\right)$ are incoming or outgoing. For example, if the boundary in question is the outer radial boundary, and the flow in the radial 
direction is outgoing and subsonic, then the $L_{1}$ wave is incoming (velocity $=v-c<0$ ) and the $L_{2}-L_{4}$ waves are all outgoing. At this point, the amplitude of the incoming waves are set to zero, giving the boundary condition:

$$
\begin{gathered}
\mathrm{L}_{1}=0 \\
\mathrm{~L}_{2}=(\mathrm{v})\left(\mathrm{c}^{2} \rho_{\mathrm{r}}-\mathrm{p}_{\mathrm{r}}\right) \\
\mathrm{L}_{3}=(\mathrm{v})\left(\mathrm{u}_{\mathrm{r}}\right) \\
\mathrm{L}_{4}=(\mathrm{v}+\mathrm{c})\left(\mathrm{p}_{\mathrm{r}}+\rho \mathrm{cv} \mathrm{r}_{\mathrm{r}}\right)
\end{gathered}
$$

and the time derivatives for the boundary points are calculated using Eq. (35).

The boundary conditions for the axial direction are obtained in a similar manner. After grouping the radial derivatives with the source term, Eq. (18) becomes

$$
Q_{t}+B Q_{x}+N=0
$$

The eigenvalues and eigenvectors of B are computed, and a new similarity transform is defined:

$$
\hat{\mathrm{M}}=\mathrm{T}^{-1} \mathrm{BT}=\left[\begin{array}{cccc}
\mu_{1} & 0 & 0 & 0 \\
0 & \mu_{2} & 0 & 0 \\
0 & 0 & \mu_{3} & 0 \\
0 & 0 & 0 & \mu_{4}
\end{array}\right]
$$

This time, the eigenvalues are:

$$
\begin{gathered}
\mu_{1}=u-c \\
\mu_{2}=u \\
\mu_{3}=u \\
\mu_{4}=u+c
\end{gathered}
$$

and the left eigenvectors are:

$$
m_{1}=\left\{\begin{array}{c}
0 \\
-\rho c \\
0 \\
1
\end{array}\right\}, m_{2}=\left\{\begin{array}{c}
c^{2} \\
0 \\
0 \\
-1
\end{array}\right\}, m_{3}=\left\{\begin{array}{l}
0 \\
0 \\
1 \\
0
\end{array}\right\}, m_{4}=\left\{\begin{array}{c}
0 \\
p c \\
0 \\
1
\end{array}\right\}
$$

In the axial direction, Eq. (33) become 


$$
\begin{gathered}
M_{1}=(u-c)\left(p_{x}-\rho c u_{x}\right) \\
M_{2}=(u)\left(c^{2} \rho_{x}-p_{x}\right) \\
M_{3}=(u)\left(v_{x}\right) \\
M_{4}=(u+c)\left(p_{x}+\rho c u_{x}\right)
\end{gathered}
$$

and Eq. (37) may be rewritten as:

$$
\begin{aligned}
p_{t}-\rho c u_{t} & =-M_{1}-N_{4}+\rho c N_{2}=E_{1} \\
c^{2} \rho_{t}-p_{t} & =-M_{2}-c^{2} N_{1}+N_{4}=E_{2} \\
v_{t} & =-M_{3}-N_{3}=E_{3} \\
p_{t}+\rho c u_{t} & =-M_{4}-N_{4}-\rho c N_{2}=E_{4}
\end{aligned}
$$

The boundary conditions are evaluated as before, and the time derivatives are computing using:

$$
\begin{gathered}
\rho_{\mathrm{t}}=\frac{1}{\mathrm{c}^{2}}\left(\mathrm{E}_{2}+\frac{1}{2}\left(\mathrm{E}_{1}+\mathrm{E}_{4}\right)\right) \\
\mathrm{u}_{\mathrm{t}}=\frac{1}{2 \rho \mathrm{c}}\left(\mathrm{E}_{4}-\mathrm{E}_{1}\right) \\
\mathrm{v}_{\mathrm{t}}=\mathrm{E}_{3} \\
\mathrm{p}_{\mathrm{t}}=\frac{1}{2}\left(\mathrm{E}_{1}+\mathrm{E}_{4}\right)
\end{gathered}
$$

\section{GILES (1990)}

Giles $^{8}$ derived boundary conditions based on Fourier analysis of the linearized Euler equations (LEE). Consider the unsteady part of the flow to be small disturbances, denoted by ', superimposed on a constant mean flow U, V. The axisymmetric LEE can be written as:

$$
\mathrm{Q}_{\mathrm{t}}^{\prime}+\mathrm{AQ}_{\mathrm{r}}^{\prime}+\mathrm{BQ}_{\mathrm{X}}^{\prime}+\mathrm{C}^{\prime}=0
$$

where

$$
\begin{gathered}
Q=\left\{\begin{array}{l}
\rho^{\prime} \\
u^{\prime} \\
y^{\prime} \\
p^{\prime}
\end{array}\right\} \\
A=\left[\begin{array}{cccc}
V & 0 & \bar{\rho} & 0 \\
0 & V & 0 & 0 \\
0 & 0 & V & \overline{\bar{\rho}} \\
0 & 0 & \bar{c}^{2} \bar{\rho} & V
\end{array}\right]
\end{gathered}
$$




$$
\mathrm{B}=\left[\begin{array}{cccc}
\mathrm{U} & \bar{\rho} & 0 & 0 \\
0 & \mathrm{U} & 0 & \overline{\bar{\rho}} \\
0 & 0 & \mathrm{U} & 0 \\
0 & \overline{\mathrm{c}}^{2} \bar{\rho} & 0 & \mathrm{U}
\end{array}\right]
$$

and

$$
C^{\prime}=\frac{1}{r}\left\{\begin{array}{c}
-\bar{\rho} v \\
0 \\
0 \\
-\bar{c}^{2} \bar{\rho} v
\end{array}\right\}
$$

An overbar denotes a mean flow quantity.

Consider the disturbance to be in the wave form:

$$
Q^{\prime}(x, r, t)=q^{R} e^{i(k x+l r-\omega t)}
$$

where $\mathrm{q}^{\mathrm{R}}$ is a constant vector. Giles' basic idea is that the dispersion relation for the above linear equation can be modified to prohibit propagation for waves with group velocities directed into the computational domain. This dispersion relation is nonlinear, which implies that the boundary condition must be a nonlocal one to satisfy this condition exactly. To produce local boundary conditions, the dispersion relation is expanded in a Taylor series around the one-dimensional solution. Boundary conditions for various degrees of approximation can thus be constructed.

In the axial direction, the characteristic variables are defined as:

$$
\begin{aligned}
& r_{1}=p^{\prime}-\bar{\rho} \bar{c} u^{\prime} \\
& r_{2}=p^{\prime}-\bar{c}^{2} \rho^{\prime} \\
& r_{3}=p^{\prime}+\bar{\rho} \bar{c} u^{\prime} \\
& r_{4}=\bar{\rho} \bar{c} v^{\prime}
\end{aligned}
$$

For a subsonic outflow condition $(\mathrm{x}=\mathrm{b})$, the $\mathrm{r}_{1}$ characteristic is obtained using the Giles analysis; all others are obtained from the inner solution. The second-order Giles outflow condition is:

$$
\frac{\partial r_{1}}{\partial t}=-U\left(\frac{\partial r_{4}}{\partial r}+\frac{r_{4}}{r}\right)-V \frac{\partial r_{1}}{\partial r}
$$

For a subsonic inflow condition $(\mathrm{x}=\mathrm{a})$, the fourth-order Giles boundary condition becomes:

$$
\begin{gathered}
\frac{\partial r_{2}}{\partial t}=-V \frac{\partial r_{2}}{\partial r} \frac{\partial r_{3}}{\partial t}=-\frac{(\bar{c}-U)}{2}\left(\frac{\partial r_{4}}{\partial r}+\frac{r_{4}}{r}\right)-V \frac{\partial r_{3}}{\partial r} \\
\frac{\partial r_{4}}{\partial t}=-V\left(\frac{\partial r_{4}}{\partial r}+\frac{r_{4}}{r}\right)-\frac{(\bar{c}+U)}{2} \frac{\partial r_{3}}{\partial r}-\frac{(\bar{c}-U)}{2} \frac{\partial r_{1}}{\partial r}
\end{gathered}
$$


Likewise, for an inflow at an outer radial boundary ( $r=\mathrm{rmax}$ ), the characteristic variables are:

$$
\begin{aligned}
& c_{1}=p^{\prime}-\bar{\rho} \bar{c} v^{\prime} \\
& c_{2}=p^{\prime}-\bar{c}^{2} \rho^{\prime} \\
& c_{3}=p^{\prime}+\bar{\rho} \bar{c} v^{\prime} \\
& c_{4}=\bar{\rho} \bar{c} u^{\prime}
\end{aligned}
$$

The subsonic inflow condition becomes:

$$
\begin{gathered}
\frac{\partial c_{1}}{\partial t}=-\frac{(\bar{c}-V)}{2} \frac{\partial c_{4}}{\partial x}-U \frac{\partial c_{1}}{\partial x} \\
\frac{\partial c_{2}}{\partial t}=-U \frac{\partial c_{2}}{\partial x} \\
\frac{\partial c_{4}}{\partial t}=-U \frac{\partial c_{4}}{\partial x}-\frac{(\bar{c}+V)}{2} \frac{\partial c_{3}}{\partial x}-\frac{(\bar{c}-V)}{2} \frac{\partial c_{1}}{\partial x}
\end{gathered}
$$

TAM AND WEBB (1993)

Tam and Webb, ${ }^{9}$ Bayliss and Turkel, ${ }^{10}$ Hagstrom and Hariharan, ${ }^{11}$ and Enquest and Majda ${ }^{12}$ considered boundary conditions based on an asymptotic analysis of the Linearized Euler Equations with constant mean flow in the x-direction:

$$
\begin{aligned}
& \mathrm{u}_{\mathrm{t}}^{\prime}+\mathrm{Uu}_{\mathrm{x}}{ }+\frac{1}{\bar{\rho}} \mathrm{p}_{\mathrm{x}}{ }_{\mathrm{x}}=0 \\
& \mathrm{v}_{\mathrm{t}}^{{ }^{\prime}}+\mathrm{Uv}_{\mathrm{x}}{ }_{\mathrm{x}}+\frac{1}{\bar{\rho}} \mathrm{p}_{\mathrm{r}}^{\prime}=0 \\
& \mathrm{p}_{{ }_{\mathrm{t}}}+\mathrm{Up}_{\mathrm{x}}{ }_{\mathrm{x}}+\bar{\rho} \overline{\mathrm{c}}^{2}\left(\mathrm{u}_{\mathrm{x}}{ }_{\mathrm{x}}+\mathrm{v}_{\mathrm{r}}^{\prime}+\frac{\mathrm{v}^{\prime}}{\mathrm{r}}\right)=0
\end{aligned}
$$

The above system of equations can be reduced to the convective wave equation for the pressure. For an outgoing wave solution at large distances, the boundary condition can be stated as:

$$
\frac{1}{V(\theta)} p_{t}^{\prime}+p_{R}^{\prime}+\frac{p^{\prime}}{R}=0
$$

where

$$
\begin{gathered}
R=\sqrt{x^{2}+r^{2}} \\
V(\theta)=\bar{c}\left[\frac{x}{R} M+\sqrt{1-\left(\frac{r}{R} M\right)^{2}}\right]
\end{gathered}
$$

and 


$$
M=\frac{U}{\bar{c}}
$$

The above form of the pressure boundary condition was obtained by Tam and Webb, but one can show that it is identical to the pressure condition obtained by Bayliss and Turkel and others.

However, Tam and Webb performed a rational asymptotic analysis for the full set of Euler equations. They showed that while the asymptotic pressure is only acoustic in nature, the velocity and density fluctuations contain both hydrodynamic and acoustic contributions. Using this, they arrived at a complete set of outflow boundary conditions which can be written in the form:

$$
\begin{gathered}
\rho_{t}^{\prime}=-U \rho_{X}^{\prime}+\frac{1}{\bar{c}^{2}}\left(p_{t}^{\prime}+U_{p_{X}^{\prime}}\right) \\
u_{t}^{\prime}=-U u_{x}^{\prime}-\frac{1}{\bar{\rho}} p_{x}^{\prime} \\
v_{t}^{\prime}=-U v_{x}^{\prime}-\frac{1}{\bar{\rho}} p_{r}^{\prime} \\
p_{t}^{\prime}=-V(\theta)\left[\frac{x}{R} p_{x}^{\prime}+\frac{r}{R} p_{r}^{\prime}+\frac{p^{\prime}}{R}\right]
\end{gathered}
$$

This boundary condition is applied at the outflow boundary; at the inflow boundaries, acoustic radiation boundary conditions may be used:

$$
Q_{t}^{\prime}=-V(\theta)\left[\frac{x}{R} Q^{\prime}{ }_{x}+\frac{r}{R} Q_{r}^{\prime}+\frac{Q^{\prime}}{R}\right]
$$

where:

$$
Q^{\prime}=\left\{\begin{array}{l}
\rho^{\prime} \\
u^{\prime} \\
v^{\prime} \\
p^{\prime}
\end{array}\right\}
$$

\section{TEST PROBLEM}

The test problem studied was that of a monopole in a uniform flow in the axial direction. The analytic solution is:

$$
\begin{gathered}
p^{\prime}(x, r, t)=\frac{\bar{\rho} S}{4 \pi D}\left[\left(1+\frac{1}{\beta^{2}}\left(M^{2}-\frac{M x}{D}\right)\right) \omega \cos (\omega \tau)-\frac{U x}{D^{2}} \sin (\omega \tau)\right] \\
u^{\prime}(x, r, t)=\frac{S}{4 \pi D}\left[\frac{1}{\beta^{2} \bar{c}}\left(M-\frac{x}{D}\right) \omega \cos (\omega \tau)-\frac{x}{D^{2}} \sin (\omega \tau)\right]
\end{gathered}
$$




$$
\begin{gathered}
v^{\prime}(x, r, t)=-\frac{S r}{4 \pi D^{2}}\left[\frac{\omega}{\bar{c}} \cos (\omega \tau)-\frac{\beta^{2}}{D} \sin (\omega \tau)\right] \\
\rho^{\prime}(x, r, t)=\frac{p^{\prime}(x, r, t)}{\bar{c}^{2}}
\end{gathered}
$$

where:

$$
\begin{gathered}
M=\frac{U}{\bar{c}} \\
\beta=\sqrt{1-M^{2}} \\
D=\sqrt{x^{2}+(\beta r)^{2}} \\
\tau=t-\frac{D-M x}{\bar{c} \beta^{2}}
\end{gathered}
$$

For the described computations,

$$
\begin{gathered}
\omega=\frac{\pi}{2} \\
S=.01 \\
M=0.5
\end{gathered}
$$

The computational grid starts at $\mathrm{r}=0.5$ and ends at $\mathrm{r}=24.5$ in the radial direction. In the axial direction, the grid covers from $-12<x<12$. The grid has $300 \times 300$ points, and is not stretched in either direction. Tests were also performed with a finer grid of $450 \times 450$ points; while the overall errors decreased, the relative performances of the boundary conditions were the same.

The point source is located at the origin. The analytic solution is specified as the initial conditions, and a CFL of 0.5 is used. With no mean flow, this gives 25 points per wavelength, and 191 time steps per cycle of oscillation.

For all computations, the analytic solution was specified at the inner radial boundary. The upstream axial boundary and the outer radial boundary were both treated as inflow boundaries, while the downstream axial boundary was treated as an outflow boundary.

\section{IMPLEMENTATION OF BOUNDARY CONDITIONS}

\section{Thompson}

The Thompson boundary condition was implemented in four ways: two were second-order accurate in time, while the remaining two were first order accurate in time. The axial outflow boundary will be used to illustrate the various implementations of the Thompson boundary condition.

Due to the split operators used by the code, the implementation of the Thompson boundary conditions was greatly simplified. The axial operator used by the code is defined by Eq. (12) as:

$$
\frac{\partial \tilde{Q}}{\partial t}+\frac{\partial \tilde{F}}{\partial x}=0
$$


Thus, Eq. (37) becomes:

$$
\mathrm{Q}_{\mathrm{t}}+\mathrm{BQ} \mathrm{x}=0
$$

since the radial derivatives and the source term do not appear in the axial operator. Using these definitions, Eq. (42) is rewritten as:

$$
\begin{gathered}
\mathrm{p}_{\mathrm{t}}-\rho \mathrm{cu} \mathrm{t}=-\mathrm{M}_{1}=\mathrm{U}_{1} \\
\mathrm{c}^{2} \rho_{\mathrm{t}}-\mathrm{p}_{\mathrm{t}}=-\mathrm{M}_{2}=\mathrm{U}_{2} \\
\mathrm{v}_{\mathrm{t}}=-\mathrm{M}_{3}=\mathrm{U}_{3} \\
\mathrm{p}_{\mathrm{t}}+\rho_{\mathrm{c}}=-\mathrm{M}_{4}=\mathrm{U}_{4}
\end{gathered}
$$

Using Eq. (73), the amplitude of the characteristic waves $\mathrm{U}_{1}-\mathrm{U}_{4}$ can be computed using either time or space derivatives.

At the corners, the boundary conditions for both grid faces are applied.

Thompson split/time (Th2t). The first implementation of the Thompson boundary condition used the time derivatives to compute the amplitudes of the characteristic waves. To preserve the code's second-order time accuracy, the boundary conditions were applied at the end of both the predictor and corrector sweeps in each direction.

Thompson split/space (Th2s). This variant used the spatial derivatives to compute the amplitude of the characteristic waves. The spatial derivatives were computed in the same manner as shown in Eq. (14) and (15), using extrapolation when required. Note that the derivatives are computed using the primitive variables, not the conserved quantities. Again, the boundary conditions are applied at the end of the predictor and corrector sweeps in each direction.

Thompson/space (Th1s). This version also used the spatial derivatives to compute the amplitude of the characteristic waves. This time, however, the boundary conditions were only applied at the end of the corrector sweep, using fourth-order accurate one-sided differences to evaluate the spatial derivatives at the old time level. First-order accurate time derivatives are used to update the flow variables at the boundaries.

Thompson/time (Th1t). The time derivatives were used to compute the amplitude of the characteristic waves. Again, the boundary conditions were only applied at the end of the corrector sweep, using first-order accurate time derivatives.

$\underline{\text { Giles }}$

Two implementations of the Giles boundary conditions were performed, the first was first-order accurate in time, while the second was second-order accurate in time.

Giles (G1). In this version, the Giles boundary condition is implemented at the end of each time step, after both the radial and axial operators are applied. The spatial derivatives are computed using fourth-order accurate one-sided differences, with the flow variables from the old time level. The time derivatives are computed using first-order backward differences.

Giles split (G2). Next, the Giles boundary condition was split into axial and radial operators. In this formulation, the boundary conditions are applied at the end of each predictor and corrector sweep, returning second order accuracy in time.

The splitting procedure for the inflow boundary is described as an example of the methodology used. Eq. (50) gives the characteristic variables: 


$$
\begin{gathered}
r_{1}=p^{\prime}-\bar{\rho} \bar{c} u^{\prime} \\
r_{2}=p^{\prime}-\bar{c}^{2} \rho^{\prime} \\
r_{3}=p^{\prime}+\bar{\rho} \bar{c} u^{\prime} \\
r_{4}=\bar{\rho} \bar{c} v^{\prime}
\end{gathered}
$$

The total Giles boundary condition for the axial inflow is given by Eq. (52):

$$
\begin{gathered}
\frac{\partial r_{2}}{\partial t}=-V \frac{\partial r_{2}}{\partial r} \\
\frac{\partial r_{3}}{\partial t}=-\frac{(\bar{c}-U)}{2}\left(\frac{\partial r_{4}}{\partial r}+\frac{r_{4}}{r}\right)-V \frac{\partial r_{3}}{\partial r} \\
\frac{\partial r_{4}}{\partial t}=-V\left(\frac{\partial r_{4}}{\partial r}+\frac{r_{4}}{r}\right)-\frac{(\bar{c}+U)}{2} \frac{\partial r_{3}}{\partial r}-\frac{(\bar{c}-U)}{2} \frac{\partial r_{1}}{\partial r}
\end{gathered}
$$

Equation (75) is split into axial and radial operators, to be applied during the axial and radial sweeps, respectively. Since there are no axial derivatives, the boundary condition that is applied during the axial sweep is:

$$
\begin{aligned}
& \frac{\partial r_{2}}{\partial t}=0 \\
& \frac{\partial r_{3}}{\partial t}=0 \\
& \frac{\partial r_{4}}{\partial t}=0
\end{aligned}
$$

During the radial sweeps, the boundary condition is:

$$
\begin{gathered}
\frac{\partial r_{2}}{\partial t}=-V \frac{\partial r_{2}}{\partial r} \\
\frac{\partial r_{3}}{\partial t}=-\frac{(\bar{c}-U)}{2}\left(\frac{\partial r_{4}}{\partial r}+\frac{r_{4}}{r}\right)-V \frac{\partial r_{3}}{\partial r} \\
\frac{\partial r_{4}}{\partial t}=-V\left(\frac{\partial r_{4}}{\partial r}+\frac{r_{4}}{r}\right)-\frac{(\bar{c}+U)}{2} \frac{\partial r_{3}}{\partial r}-\frac{(\bar{c}-U)}{2} \frac{\partial r_{1}}{\partial r}
\end{gathered}
$$

The spatial derivatives were calculated from the primitive variables employing the differencing method used by the inner code. Extrapolation of the primitive variables was used when necessary.

The axial outflow and outer radial inflow boundary conditions are formulated in a similar way.

Both Giles boundary condition formulations were unstable when applied to corner points. Since the performance of the boundary conditions at the corner points wasn't a major area of interest, the analytic solution was specified at the corner points for these calculations. To ensure that this was not causing errors of its own, a special corner treatment based on rotated 1-D characteristics was also tested; the performance of the Giles boundary condition was insensitive to the choice of corner treatments. 


\section{Tam and Webb}

In their original implementation of this boundary condition, Tam and Webb used a 'boundary region' of two points instead of one at each edge of the grid. No extrapolation was used in computing the spatial derivatives; this required constructing stencils which only used variables at grid points in the computational domain. This is described fully in Ref. 9. To remain consistent, the Tam and Webb boundary condition was coded using only one boundary point. For comparison, a two point 'boundary region' variant was also tested; the results are equivalent. Two implementations of the Tam and Webb boundary conditions were performed.

Tam and Webb (TW1). In this version, the Tam and Webb boundary condition is implemented at the end of each time step, after both the radial and axial operators are applied. The spatial derivatives are computed using fourth-order accurate one-sided differences, with the flow variables from the old time level. The time derivatives are computed using first-order backward differences.

Tam and Webb split (TW2). Next, the Tam and Webb boundary condition was split into axial and radial operators. In this formulation, the boundary conditions are applied during each predictor and corrector sweep, retaining second order accuracy in time. The spatial derivatives were calculated from the primitive variables employing the differencing method used by the inner code. Extrapolation of the primitive variables was performed when necessary.

Since the Tam and Webb outflow condition only corrects the pressure, the original code is used to compute the other flow variables. The pressure is computed using:

$$
\mathrm{p}_{\mathrm{t}}^{\prime}=-\mathrm{V}(\theta)\left[\frac{\mathrm{x}}{\mathrm{R}} \mathrm{p}_{\mathrm{x}}^{\prime}\right]
$$

for the axial sweep, and:

$$
\mathrm{p}_{\mathrm{t}}^{\prime}=-\mathrm{V}(\theta)\left[\frac{\mathrm{r}}{\mathrm{R}} \mathrm{p}_{\mathrm{r}}^{\prime}+\frac{\mathrm{p}^{\prime}}{\mathrm{R}}\right]
$$

for the radial sweep.

For the inflow conditions at the other boundaries, the radiation boundary conditions are used. These are split as:

$$
Q_{t}^{\prime}=-V(\theta)\left[\frac{x}{R} Q_{x}^{\prime}\right]
$$

for the axial sweep, and:

$$
Q_{t}^{\prime}=-V(\theta)\left[\frac{r}{R} Q_{r}^{\prime}+\frac{Q^{\prime}}{R}\right]
$$

for the radial sweep.

$Q^{\prime}$ is defined in Eq. (64).

\section{RESULTS}

Four points were chosen in the acoustic field to evaluate the various boundary conditions at. The first point (P1) was located at $(x, r)=(-8.1,20.5)$. The second point $(\mathrm{P} 2)$ was at $(8.1,20.5)$. These points illustrate the effect of the boundary conditions on the corners of the flowfield, where the waves are not aligned with the boundaries. The third point (P3) was located at $(0,20.5)$. With no mean flow, this point shows the effectiveness of the radial boundary conditions when the wave velocity is normal to the boundary. The fourth point (P4) was located at $(10.4,2)$. With no mean flow, this point shows the effectiveness of the axial boundary conditions when the outgoing wave velocity is normal to the boundary. Also, 
an axial line (L1) was defined at $\mathrm{r}=12.5$ to better illustrate the effect of implementation differences on the performance of the various boundary conditions. Figure 1 shows the location of these points and lines in the flowfield.

At each point, the maximum amplitude of the pressure during each cycle was computed and compared to the analytic solution. The relative pressure error was used to compare the results. It is defined as:

$$
(\text { Error })_{\text {cycle }}=\left(\frac{\left|\mathrm{P}_{\text {max }}\right|_{\text {computed }}-\left|\mathrm{P}_{\text {max }}\right|_{\text {analytic }}}{\left|\mathrm{P}_{\text {max }}\right|_{\text {analytic }}}\right)_{\text {cycle }}
$$

Again, the inner radial boundary for all computations was specified from the analytic solution.

\section{No mean flow $(M=0.0)$}

Initially, the code was run for the case of a monopole in a still medium. All quantities are identical to the mean flow case with the exception of $M=0$. While this is truly a radiation boundary, it was specified as an outflow boundary when using Thompson or Giles formulations.

Figure 2 shows the maximum pressure contours for the exact solution.

Thompson. The Thompson boundary condition was the easiest to implement, and was very robust. The only version that proved unstable was the implementation which used first-order time derivatives to compute the characteristics (Th1t). This version was always unstable due to corner problems, and was abandoned.

All three implementations were basically equivalent. Figure 3 shows the maximum pressure contours for the Th4t implementation. Figure 4 shows the performance of the various implementations on the L1 line. Figure 5 shows the performance of the Th4t implementation at the four test points.

P1 and P2 show the symmetry of the test case. The errors are the highest at this point, with values of nearly $25 \%$. P3 returns the lowest error, with $3 \%$ relative pressure error. At the lower corner, $\mathrm{P} 4$ shows $7 \%$ relative pressure error.

The difference in the $\mathrm{P} 1$ and $\mathrm{P} 3$ errors is due to the angle of incidence of the acoustic waves impinging on the boundary. When the wave velocity is normal to the boundary, the Thompson boundary condition performs best, as shown by the low errors at P3 and P4.

Giles. The Giles boundary condition was the next boundary condition that was implemented. This condition was relatively easy to implement; it was also the most complex of the three due to its mixture of time and space derivatives of the primitive variables. The Giles boundary condition was stable everywhere except at the corners; since special treatments for the corner points were not given, the analytic solution was specified at the outer corner points.

There was little difference between the performance of the G1 and G2 implementations of the Giles boundary condition; Figure 6 shows the maximum pressure contours for the G1 implementation. Figure 7 shows their performance on the L1 line. Figure 8 shows the results from the G1 version at the four test points.

The Giles outflow boundary conditions also did not perform well for this test case, with relative pressure errors at P1 and P2 of nearly $30 \%$. At P3, the error is $11 \%$; while at P4, the error is less than $0.8 \%$. The effect of the fully specified corner points on the far field error was not fully investigated.

In other tests, a test case run in which the Giles inflow boundary condition is used instead of the outflow boundary condition gave much better results, with maximum errors of less than $7 \%$.

Tam and Webb. The Tam and Webb outflow boundary condition was the last formulation to be investigated. This condition does not have a special corner treatment; the corners are computed just as the other boundary points are. The 
Tam and Webb boundary condition was stable everywhere, and relatively easy to code. Figure 9 shows the maximum pressure contours for the TW2 implementation. The second order implementation (TW2) was slightly better than the first order implementation (TW1). This is illustrated in Figure 10. Figure 11 shows the results of the TW2 run; errors at all four test points were less than $2 \%$.

Test runs were made using the radiation inflow boundary condition; performance was equivalent to that of the TW2 outflow boundary condition.

Mean Flow $(M=0.5)$

Figure 12 shows the maximum pressure contours for the exact solution.

Thompson. All three implementations were all nearly equivalent. The two second-order versions (Th2s and Th2t) returned identical results, while the stable first-order implementation (Th1s) was virtually indistinguishable. Figure 13 shows the maximum pressure contours for the Th2t implementation. Figure 14 shows the results on the L1 line from the three implementations. Figure 15 shows the results of the Th2t implementation at the four test points.

The Thompson inflow condition did not perform well. For all three versions, relative pressure errors of $70 \%$ were computed at P1. It was theorized that the low performance of the inflow boundary condition in this case was due to the errors caused by the outgoing acoustic wave velocities not being normal to the boundary.

The outflow condition performed much better; at P2, the relative pressure errors were below $20 \%$. Here, the wave velocities are nearly normal to the boundary.

The inflow condition specified at the outer radial boundary (P3) resulted in relative pressure errors of $20-30 \%$.

The Thompson boundary condition performed best at P4; the velocity of the outgoing waves were nearly normal to the boundary, and the proximity of the fully specified boundary kept numerical noise to a minimum.

In general, the Thompson boundary condition was the least accurate of the boundary conditions tested.

Giles. While the differences in the results were minor, the second-order Giles implementation (G2) slightly outperformed the first-order (G1) version. Figure 16 shows the L1 results from the two implementations. Figure 17 shows the results of the G2 run at the four test points. Figure 18 shows the maximum pressure contours for the G2 run.

The Giles inflow condition worked very well, with relative pressure errors below $3 \%$ at $\mathrm{P} 1$ and $\mathrm{P} 3$. At P4, the proximity of the fully specified boundary kept the relative pressure errors below $5 \%$.

The Giles outflow condition did not perform as well as the inflow condition, with the error at P2 nearly reaching $20 \%$.

The Giles boundary condition is the second best of the three formulations tested.

Tam and Webb. Figure 19 shows the results of the runs using the Tam and Webb boundary conditions; again, the second order implementation (TW2) was slightly better than the first order implementation (TW1). Figure 20 shows the results of the TW2 run at the four test points. Figure 21 shows the maximum pressure contours for the TW2 run.

Radiation inflow conditions were used at the upstream axial and outer radial boundaries; these resulted in errors of 5\% at $\mathrm{P} 1$, and $3 \%$ at $\mathrm{P} 3$.

The Tam and Webb outflow condition performed very well, giving errors of $1 \%$ at P2 and P4.

The Tam and Webb outflow boundary is the best of the formulations tested, resulting in lower errors than the exact boundary conditions. The only drawback to this formulation is its present restriction to uniform mean flows. 


\section{CONCLUSIONS}

Several boundary conditions for computational aeroacoustics were evaluated, namely, Giles (1990), Tam and Webb (1993), and Thompson (1987). The same discretization scheme was used to compute sound radiation from a monopole placed in a stagnant or moving stream, but with the boundary treatment as described by one of the aforementioned approaches. For each boundary condition, various implementations were tested to study the sensitivity of their performance to the implementation procedure. In general, slight improvement in the performance of each scheme can be achieved via the implementation procedure.

With the best achieved performance for each boundary treatment, the following conclusion can be drawn regarding their comparative performance:

(A) For outflow boundary treatment, the only acceptable performance was that of Tam and Webb. The performance of the other schemes might be acceptable only in special cases wherein the flow is nearly one-dimensional, perpendicular to the boundary. But even under such conditions, their performance did not surpass that of Tam and Webb.

(B) For inflow boundary treatment, Giles boundary condition was acceptable, as well as the radiation boundary condition of Tam and Webb applied to inflow treatment. The Thompson inflow boundary treatment resulted in considerable reflection near the inflow boundary.

\section{REFERENCES}

1. Mankbadi, R. R., Hayder, M. E., and Povinelli, L. A., 'Structure of Supersonic Jet Flow and Its Radiated Sound', AIAA Journal, Vol. 32, No. 5, May 1994, pp. 897-906.

2. Gottlieb, D., and Turkel, E., 'Dissipative Two-Four Method for Time Dependent Problems', Mathematics of Computation, Vol. 30, No. 136, 1976, pp. 703-723.

3. Farouk, B., Oran, E. S., and Kailasanath, K., 'Numerical Simulations fo the Structure of Supersonic Shear Layers', Physics of Fluids A, Vol. 3, No. 11, 1991, pp. 2786-2798.

4. Ragab, S. A., and Sheen, S., 'The Nonlinear Development of Supersonic Instability Waves in a Mixing Layer', Physics of Fluids A, Vol. 4, No. 3, 1991, pp. 553-566.

5. Sankar, L. N., Reddy, N. N., and Hariharan, N., 'A Comparative Study of Numerical Schemes for Aero-Acoustic Applications', FED-Vol. 147, Computational Aero- and Hydro-Acoustics, ASME 1993, p. 35-40.

6. Thompson, K.W, 'Time-Dependent Boundary Conditions for Hyperbolic Systems', J. Computational Physics, Vol. 68, Jan. 1987, p. 1-24.

7. Thompson, K.W, 'Time-Dependent Boundary Conditions for Hyperbolic Systems, II', J. Computational Physics, Vol. 89,1990, p. 439-461.

8. Giles, M.B., 'Nonreflecting Boundary Conditions for Euler Equation Calculations', AIAA Journal, Vol. 28, No. 12, Dec. 1990 , p. $2050-2058$.

9. Tam, C. K. W. and Webb, J. C., 'Dispersion-Relation-Preserving Finite Difference Schemes for Computational Acoustics', J. Computational Physics, Vol. 107, 1993, pp. 262-281.

10. Bayliss, A., and Turkel, E., 'Far Field Boundary Conditions for Compressible Flows', J. Computational Physics, Vol. 48, 1982, p. 182-199.

11. Hagstrom, T., and Haraharan, S. I., 'Far Field Expansion for Anisotropic Wave Equations', Computational Acoustics, Vol. 2, 1990.

12. Enquist, B., and Majda, A., 'Radiation Boundary Conditions for Acoustic and Elastic Wave Calculations', Communications on Pure and Applied Mathematics, Vol. 32, No. 3, 1979, pp. 3-3 


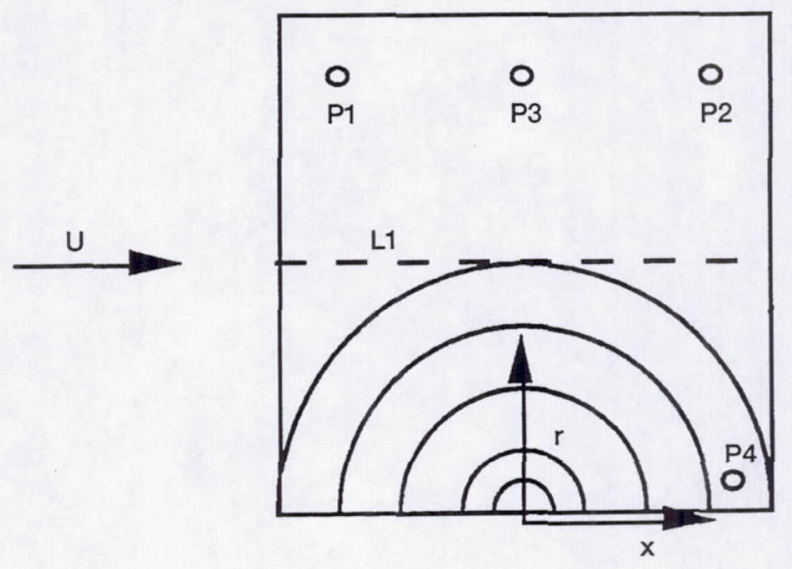

Figure 1.-Computational domain.

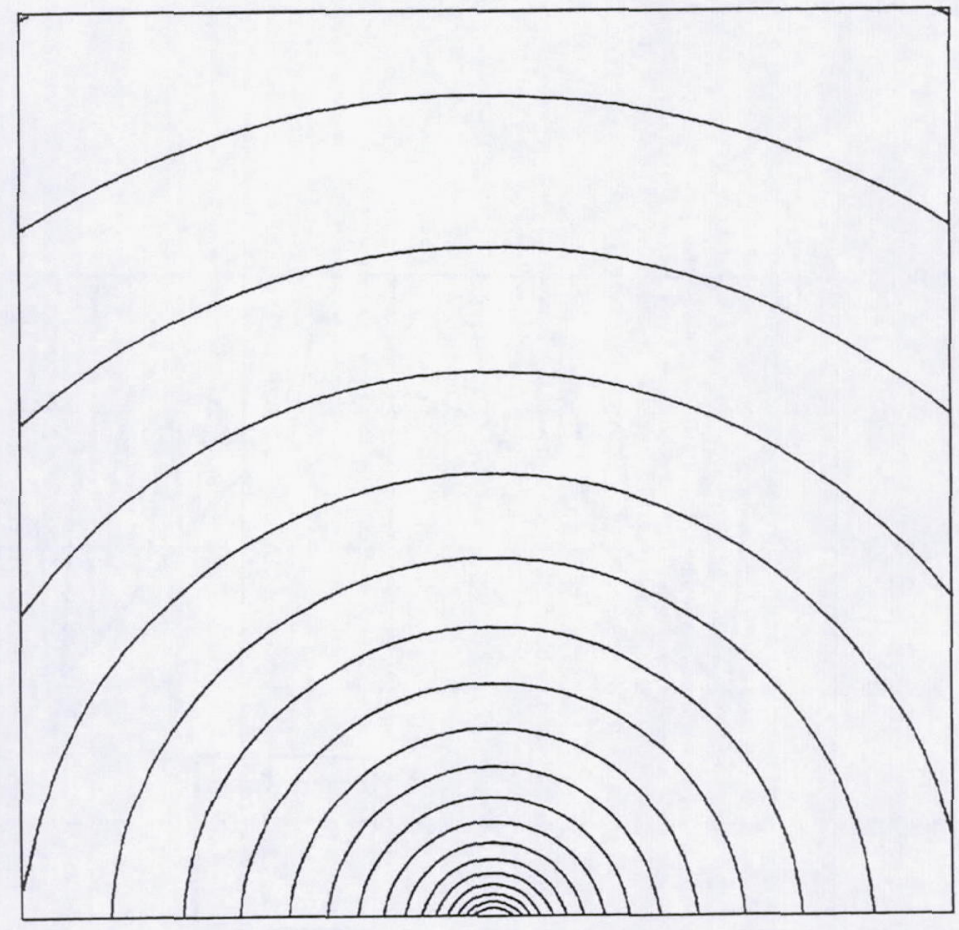

Figure 2.-Maximun pressure contours for uniform flow about a monopole ( $M=0.0$; analytical solution). 


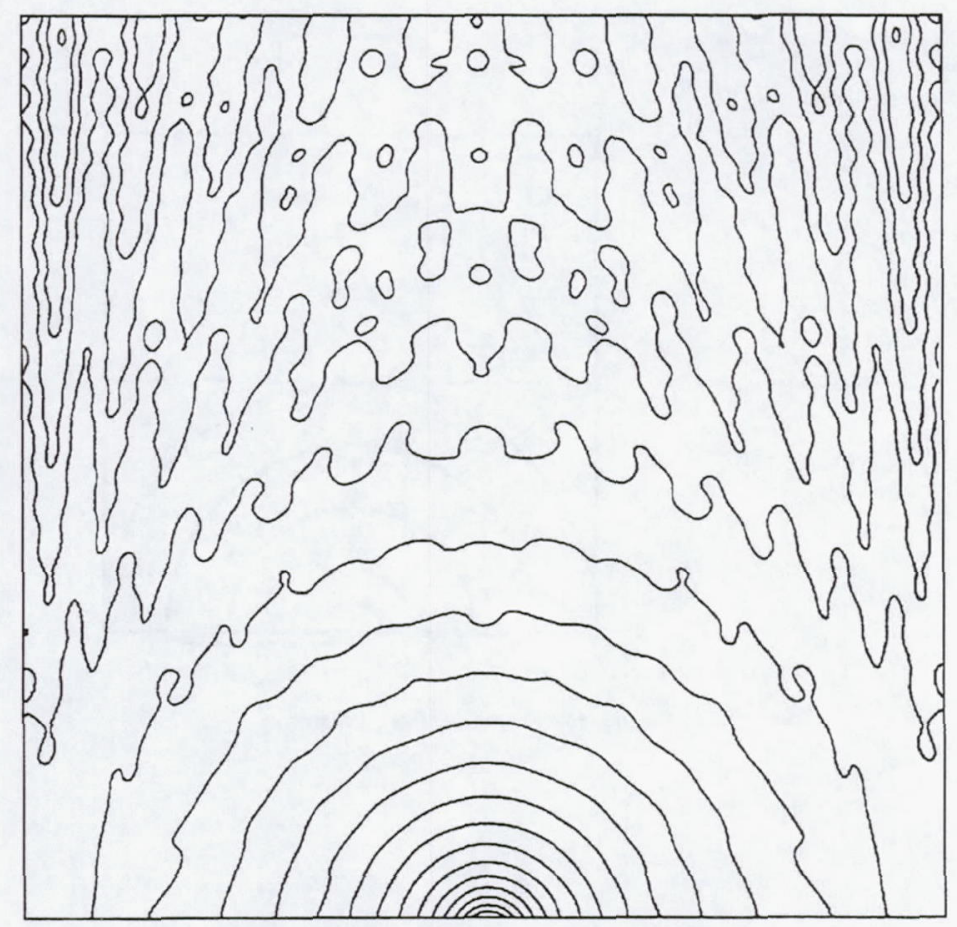

Figure 3.-Maximum pressure contours for uniform flow about a monopole ( $M=0.0$; Thompson 2nd order/time).

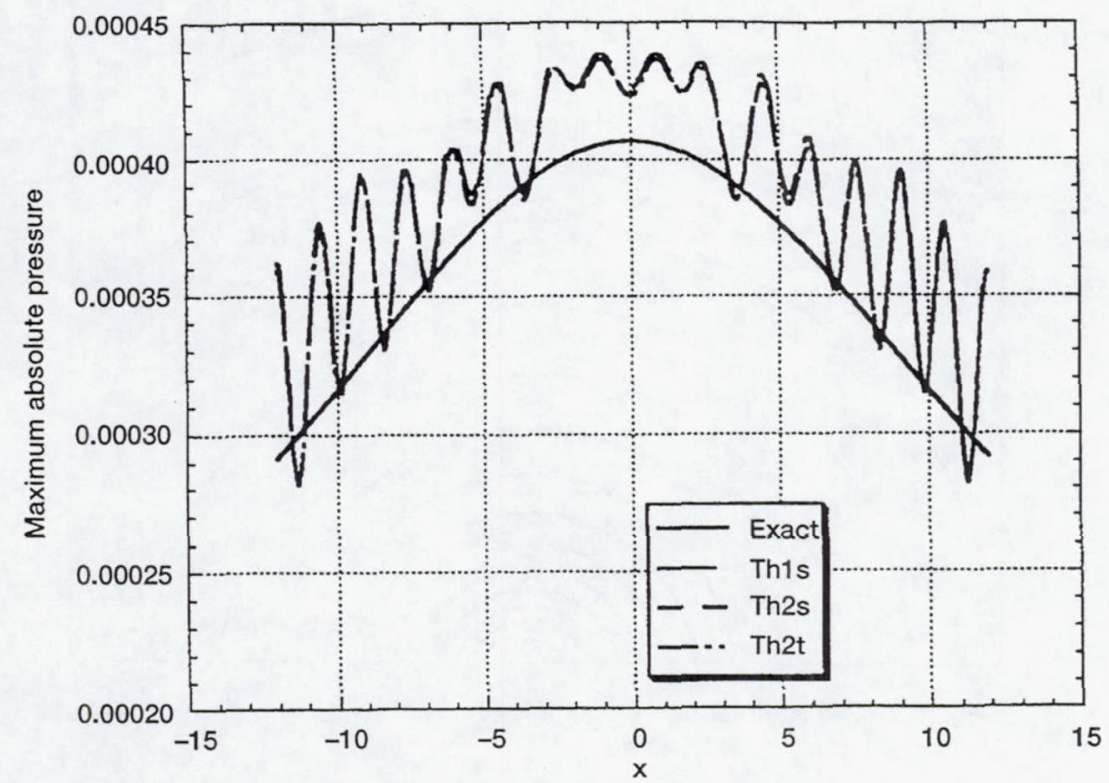

Figure 4.-Uniform flow about a monopole ( $\mathrm{M}=0.0$; Thompson). 


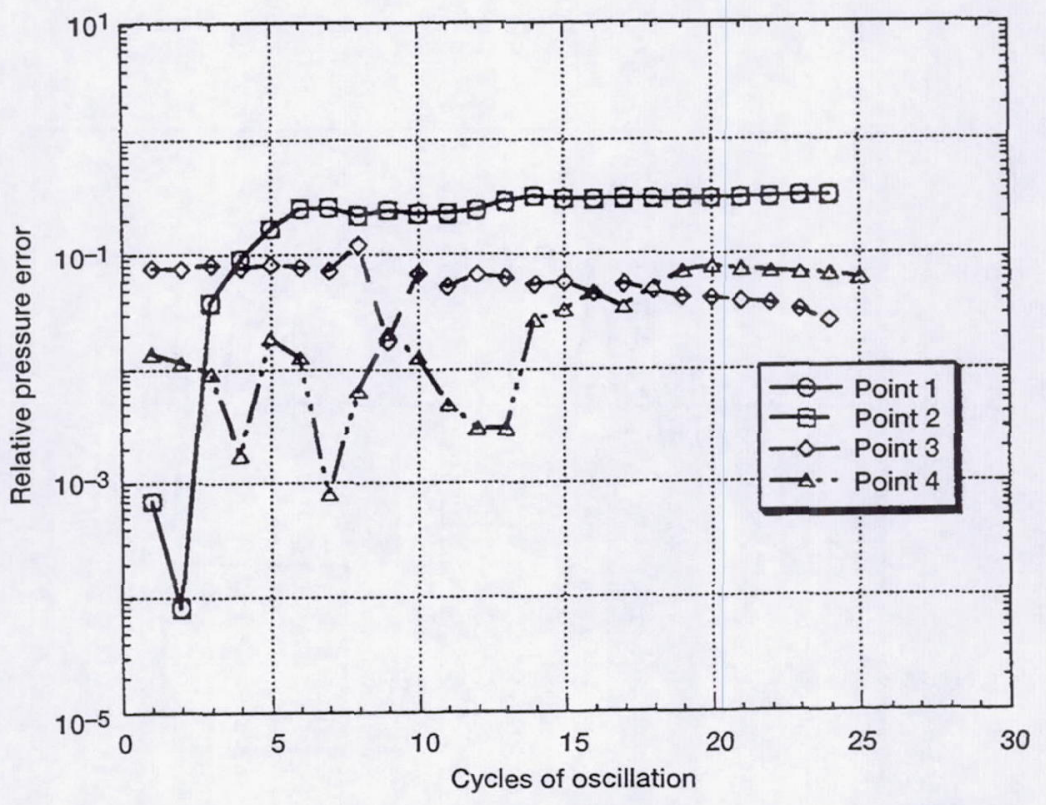

Figure 5.-Uniform flow about a monopole ( $M=0.0$; Thompson 2 nd order/time).

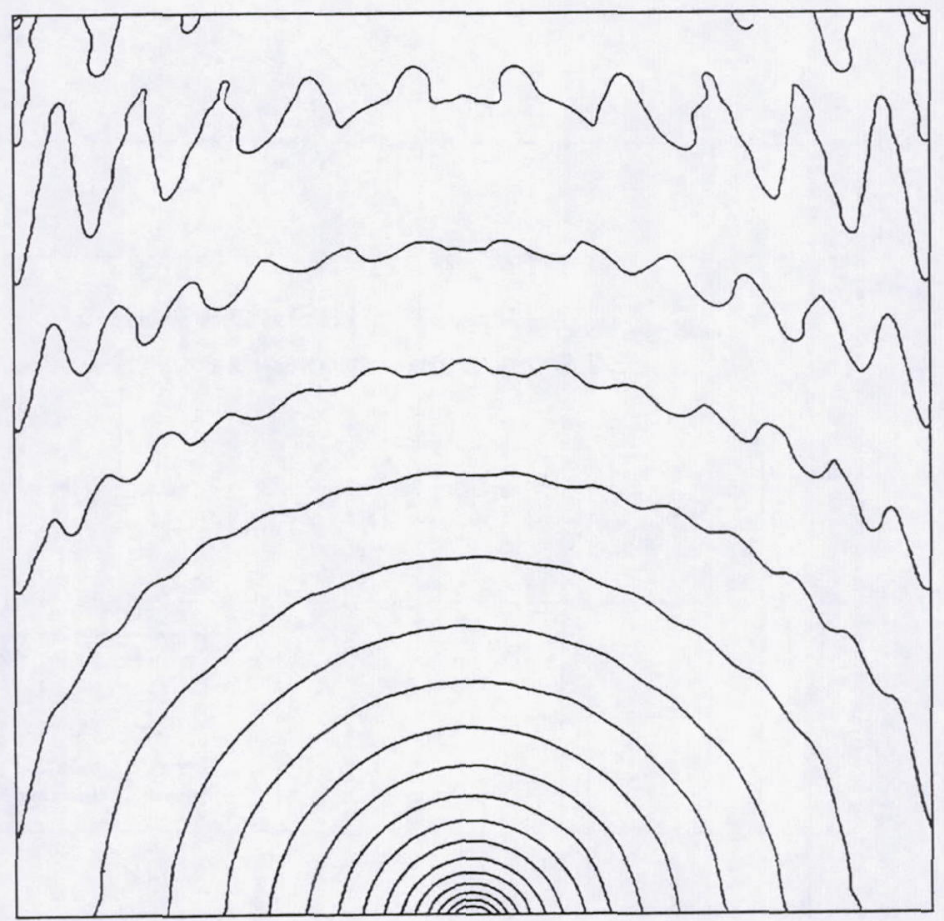

Figure 6.-Maximum pressure contours for uniform flow about a monopole ( $M=0.0$; Giles 1st order). 


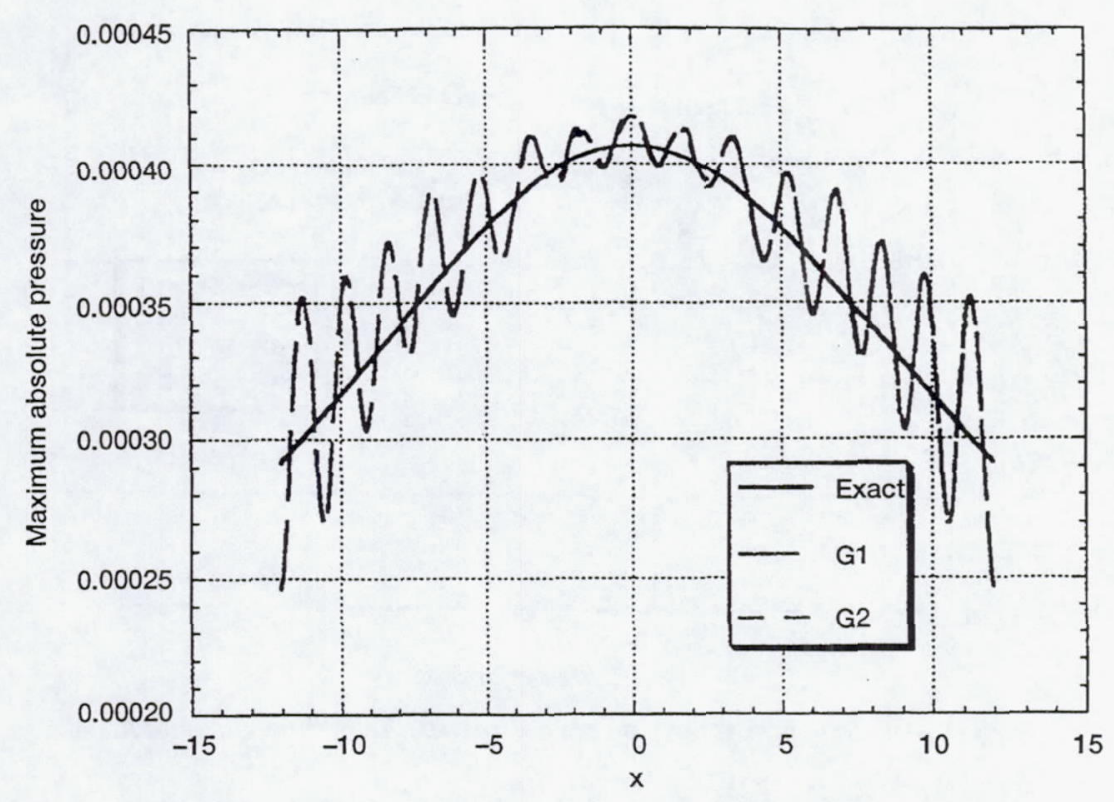

Figure 7.-Uniform flow about a monopole ( $M=0.0$; Giles).

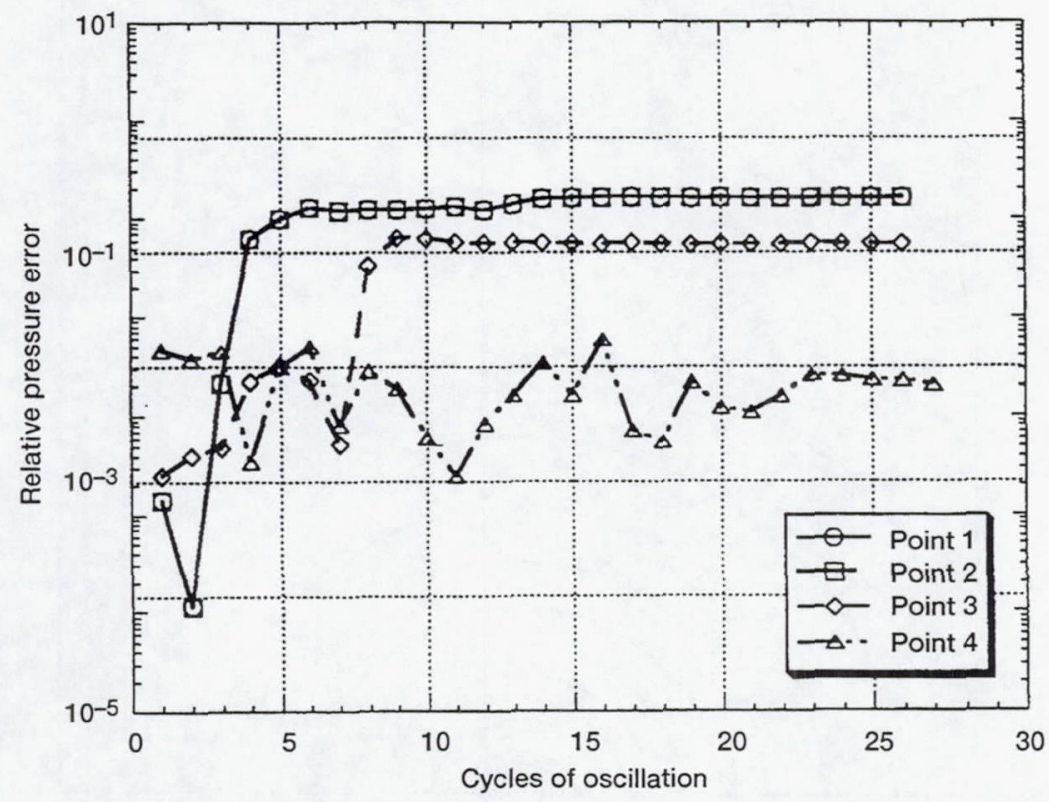

Figure 8. - Uniform flow about a monopole ( $M=0.0$; Giles 1st order). 


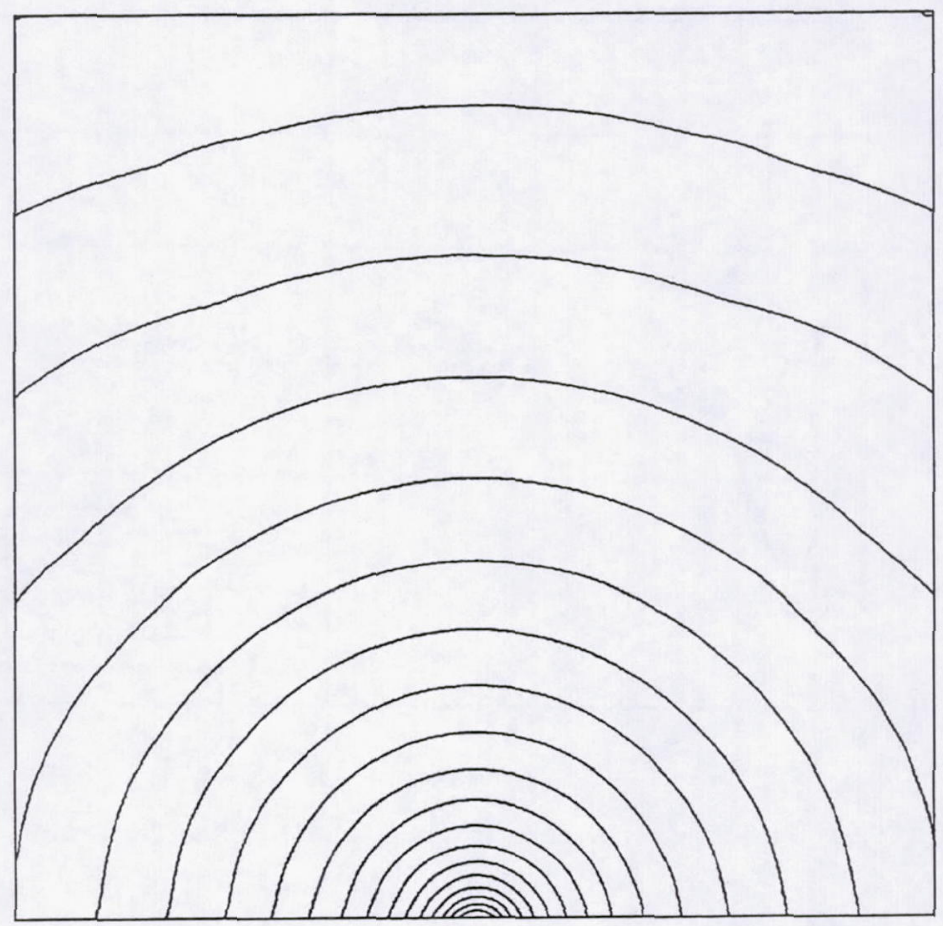

Figure 9.-Maximum pressure contours for uniform flow about a monopole ( $M=0.0$; Tam and Webb 2nd order).

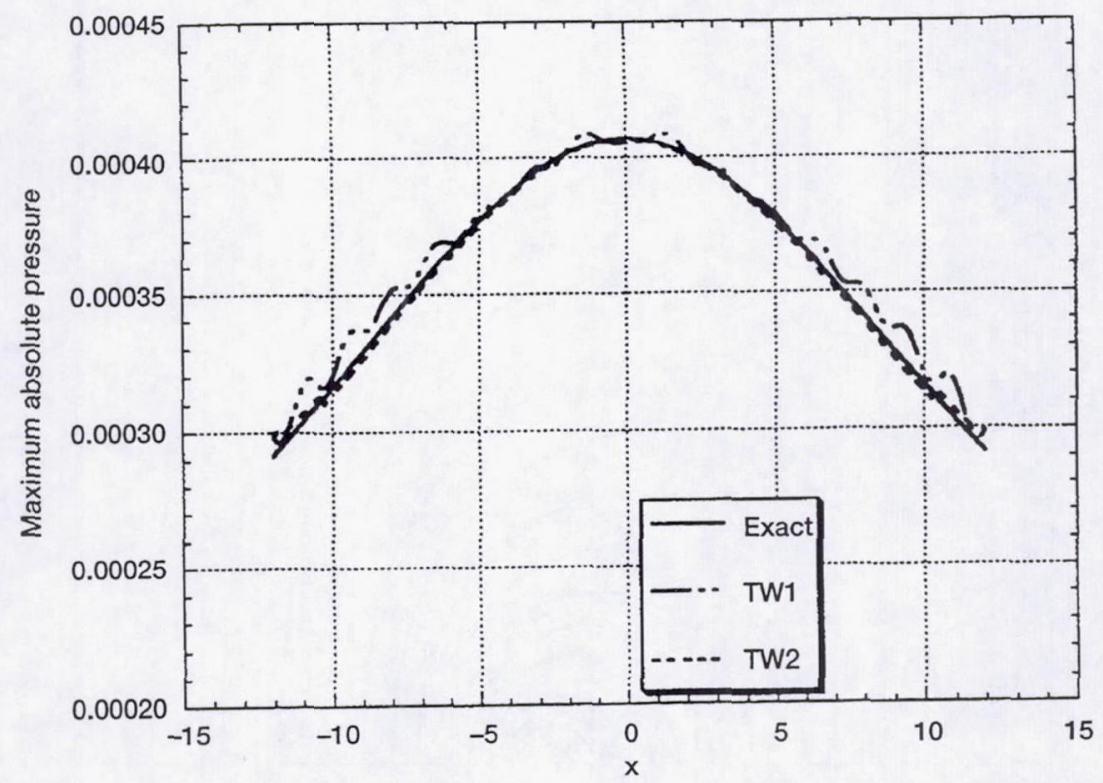

Figure 10.-Uniform flow about a monopole ( $M=0.0$; Tam and Webb). 


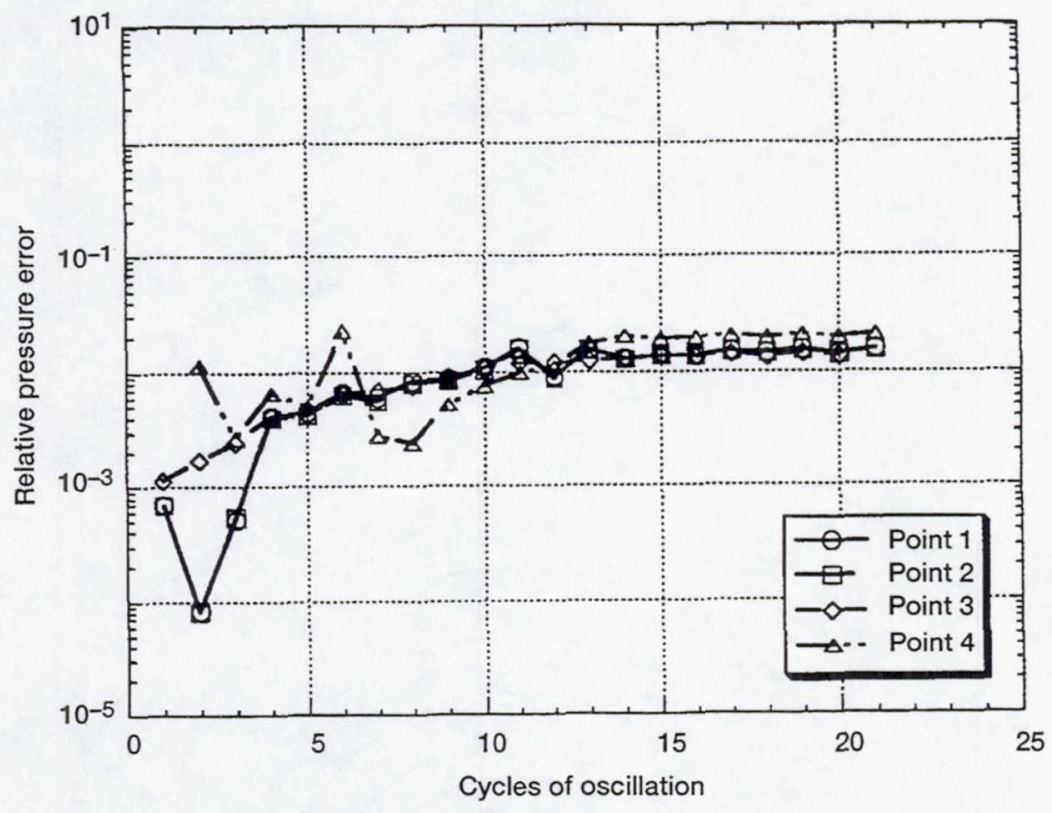

Figure 11.-Uniform flow about a monopole ( $M=0.0$; Tam 2nd order).

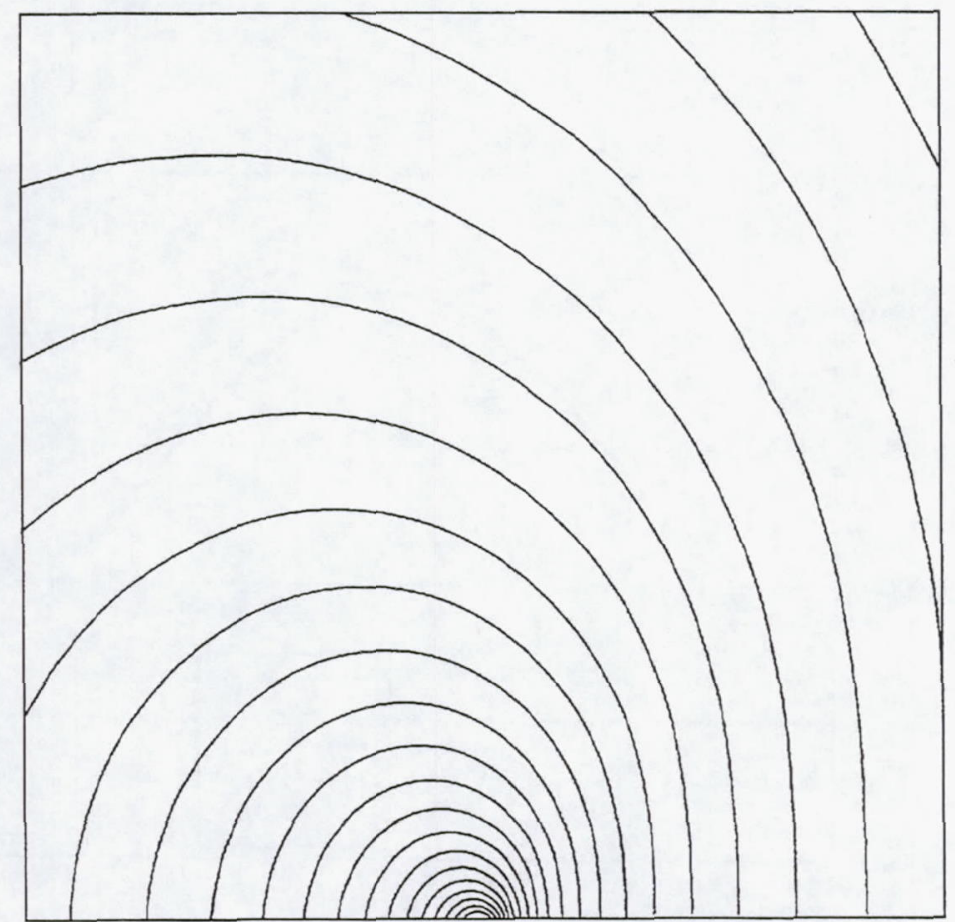

Figure 12.-Maximum pressure contours for uniform flow about a monopole ( $\mathrm{M}=0.5$; analytic solution). 


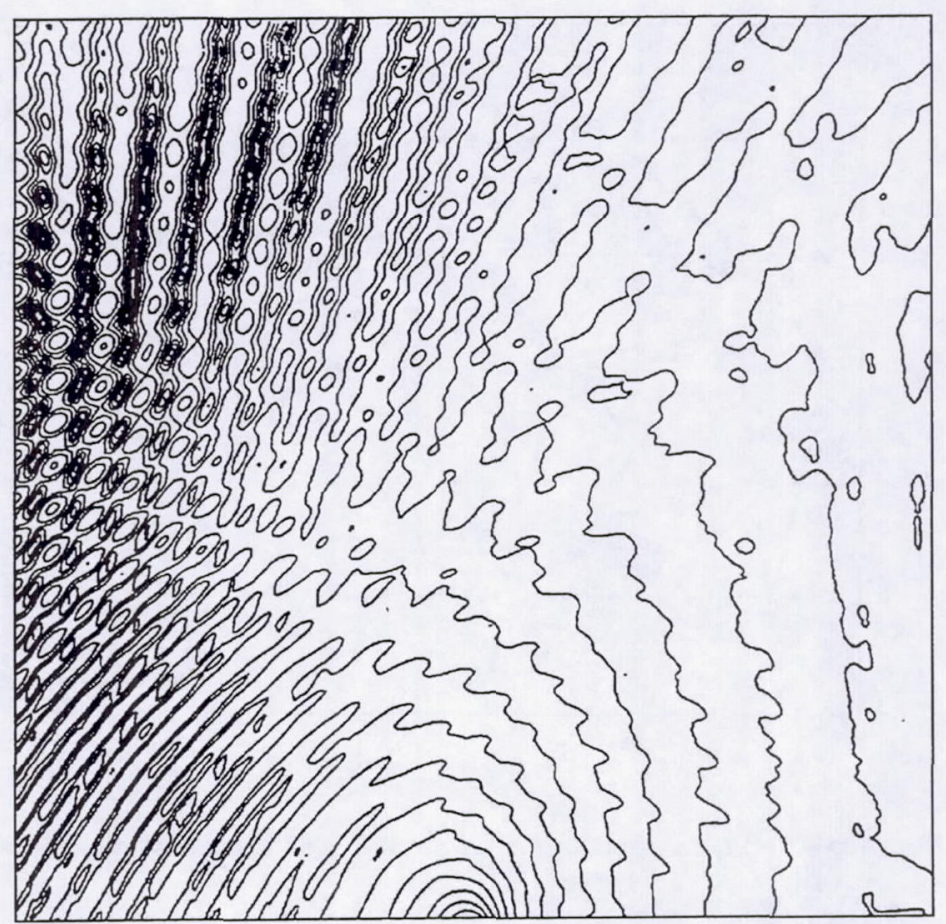

Figure 13.-Maximum pressure contours for uniform flow about a monopole ( $\mathrm{M}=0.5$; Thompson 2 nd order/time).

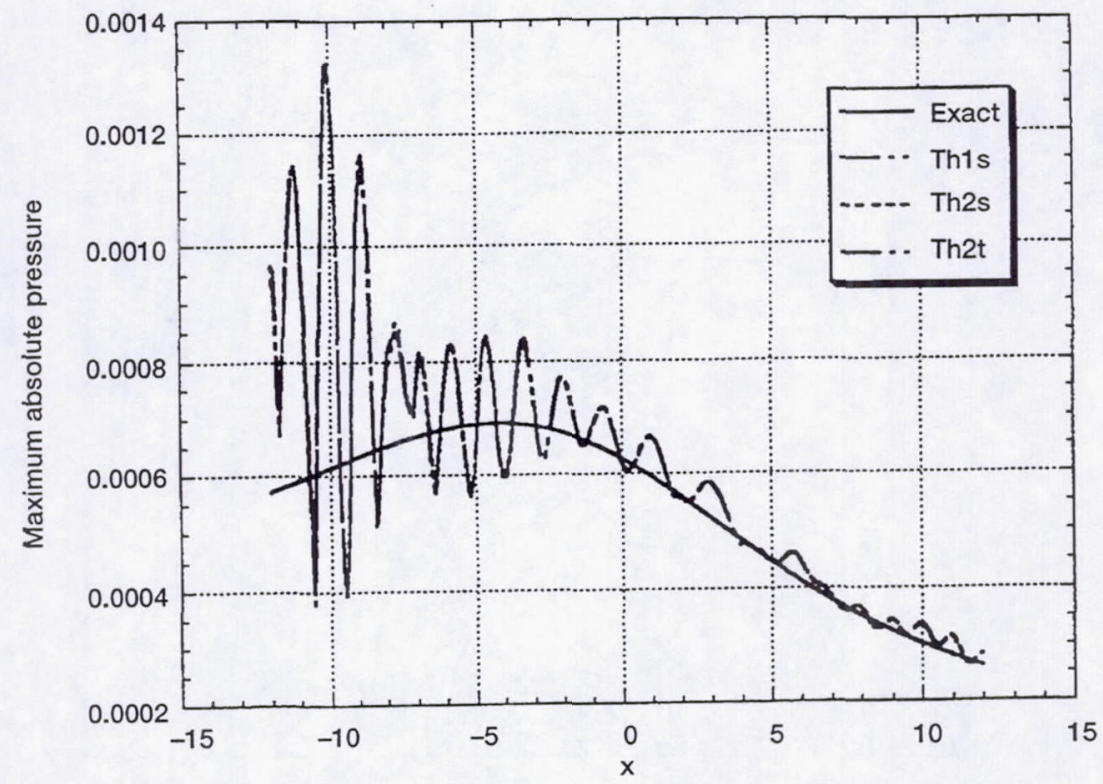

Figure 14.-Uniform flow about a monopole ( $M=0.5$; Thompson). 


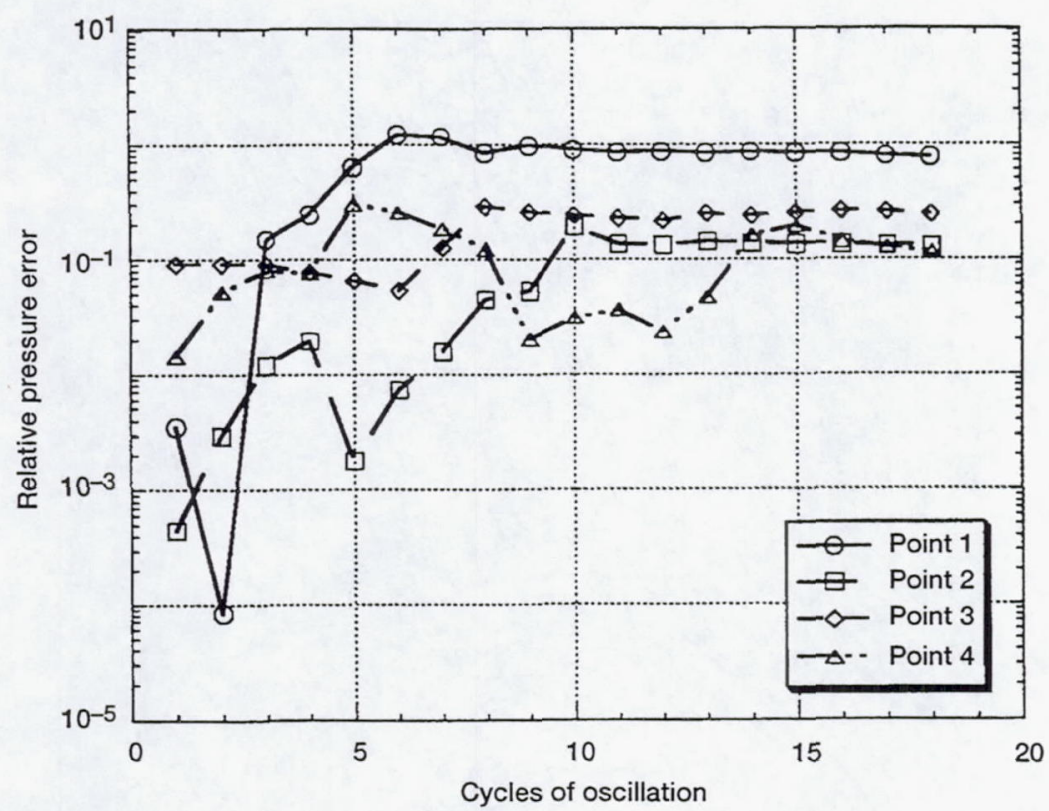

Figure 15.-Uniform flow about a monopole ( $M=0.5$; Thompson 2nd order/time).

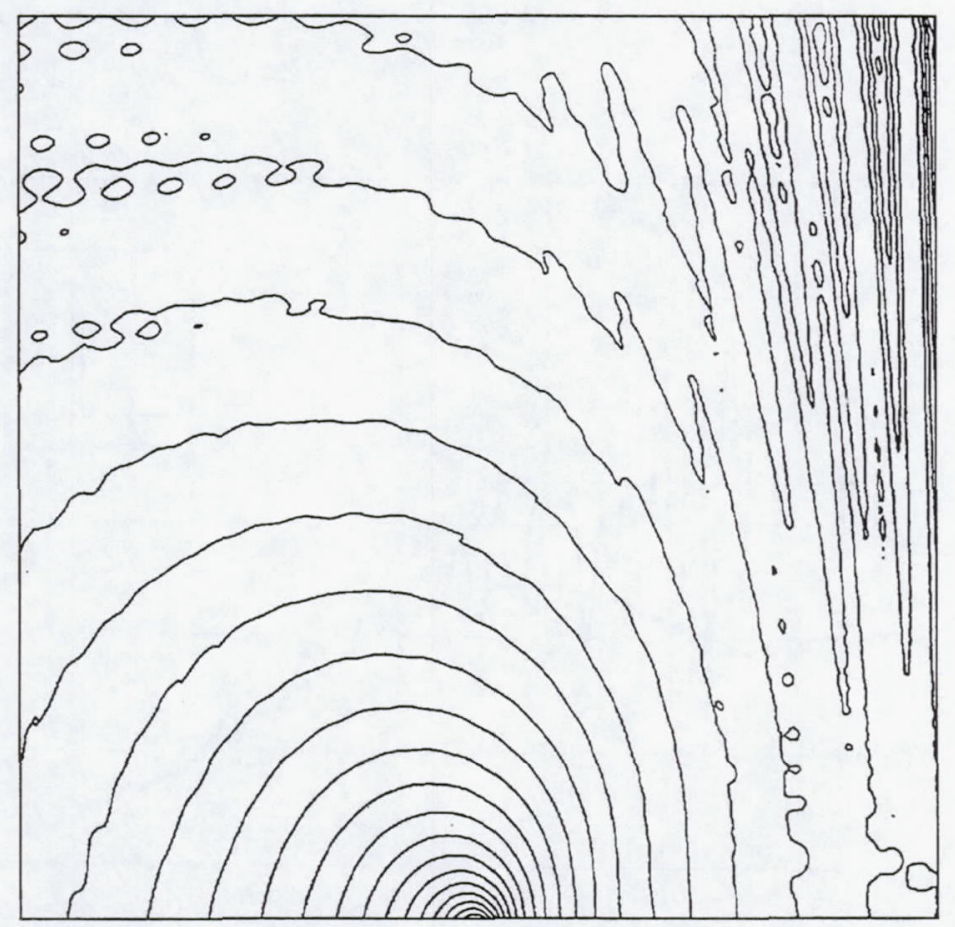

Figure 16.-Maximum pressure contours for uniform flow about a monopole ( $M=0.5$; Giles 2nd order). 


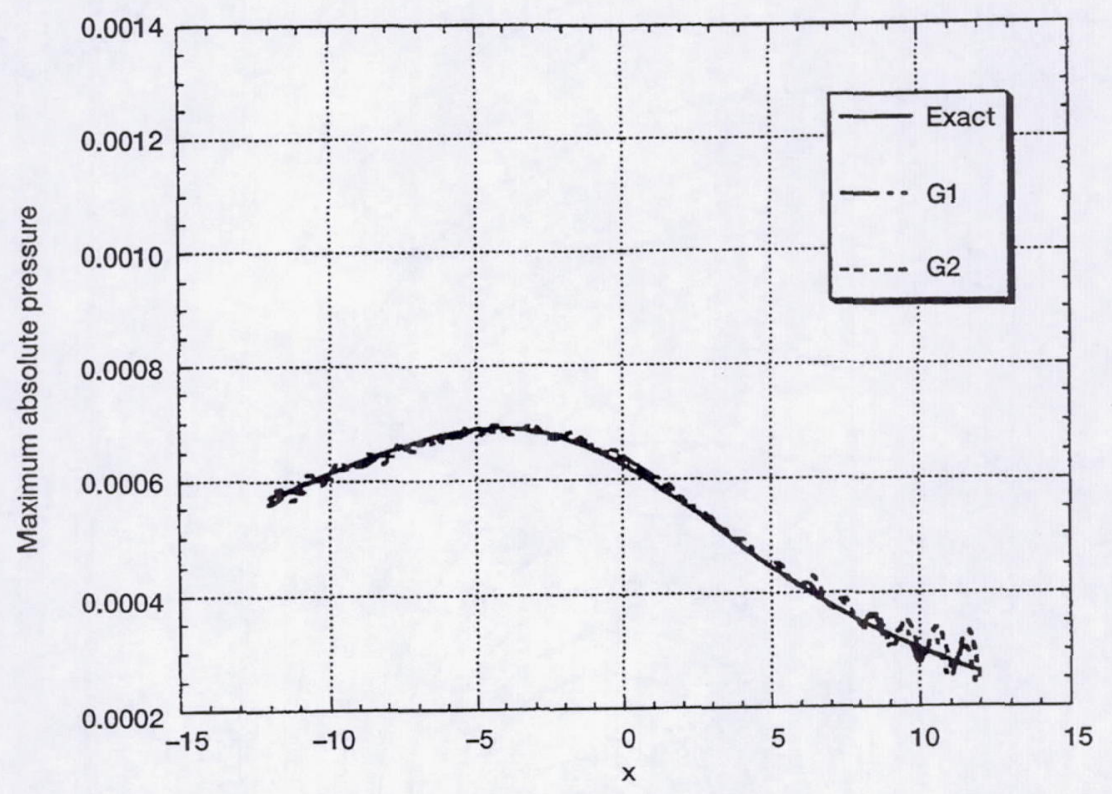

Figure 17.-Uniform flow about a monopole ( $M=0.5$; Giles).

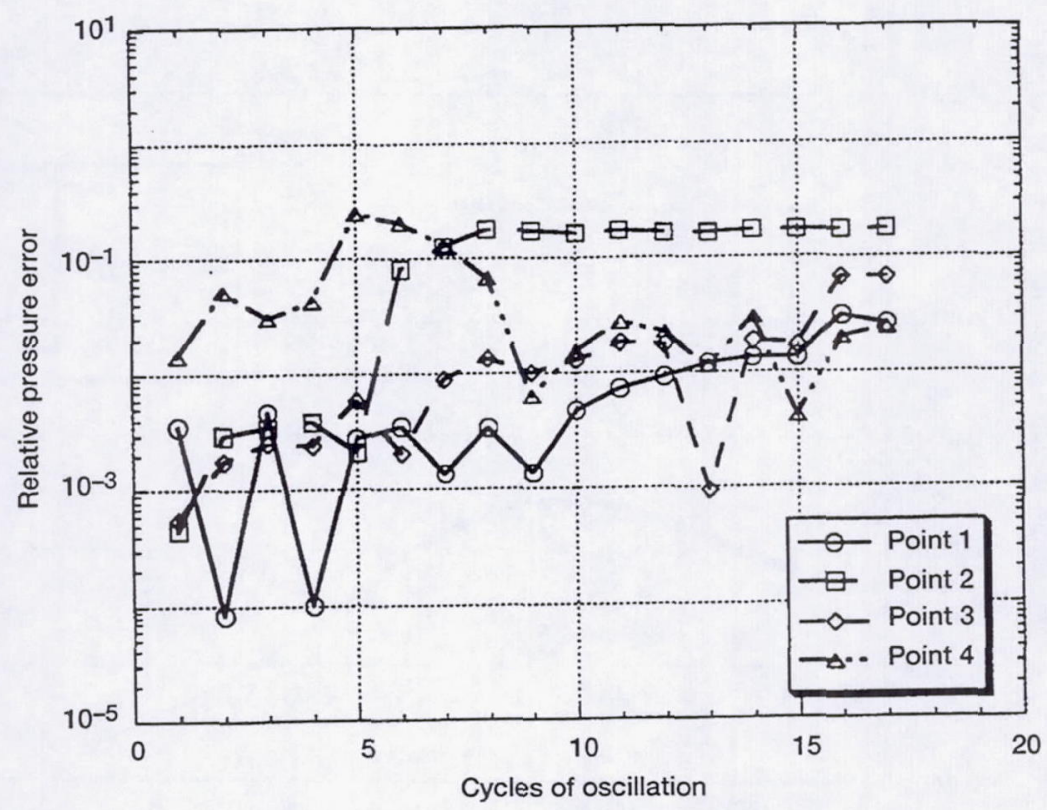

Figure 18.-Uniform flow about a monopole ( $M=0.5$; Giles 2 nd order). 


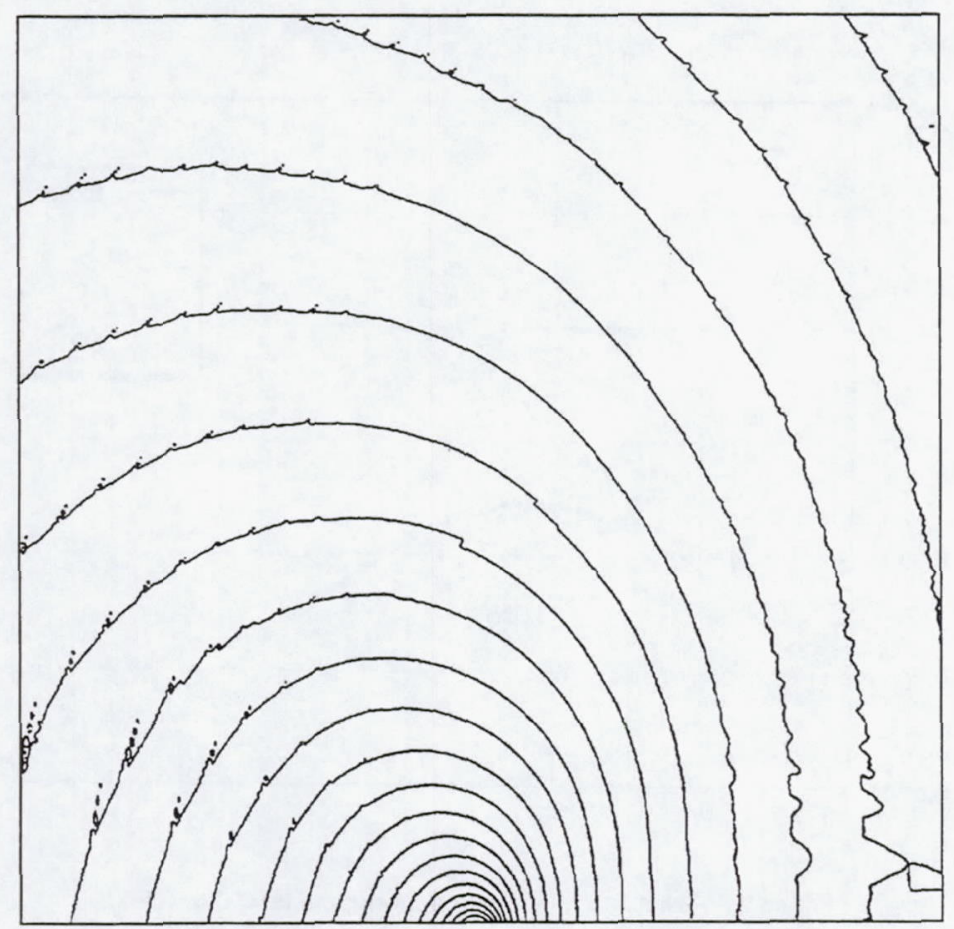

Figure 19.-Maximum pressure contours for uniform flow about a monople ( $M=0.5$; Tam and Webb 2nd order).

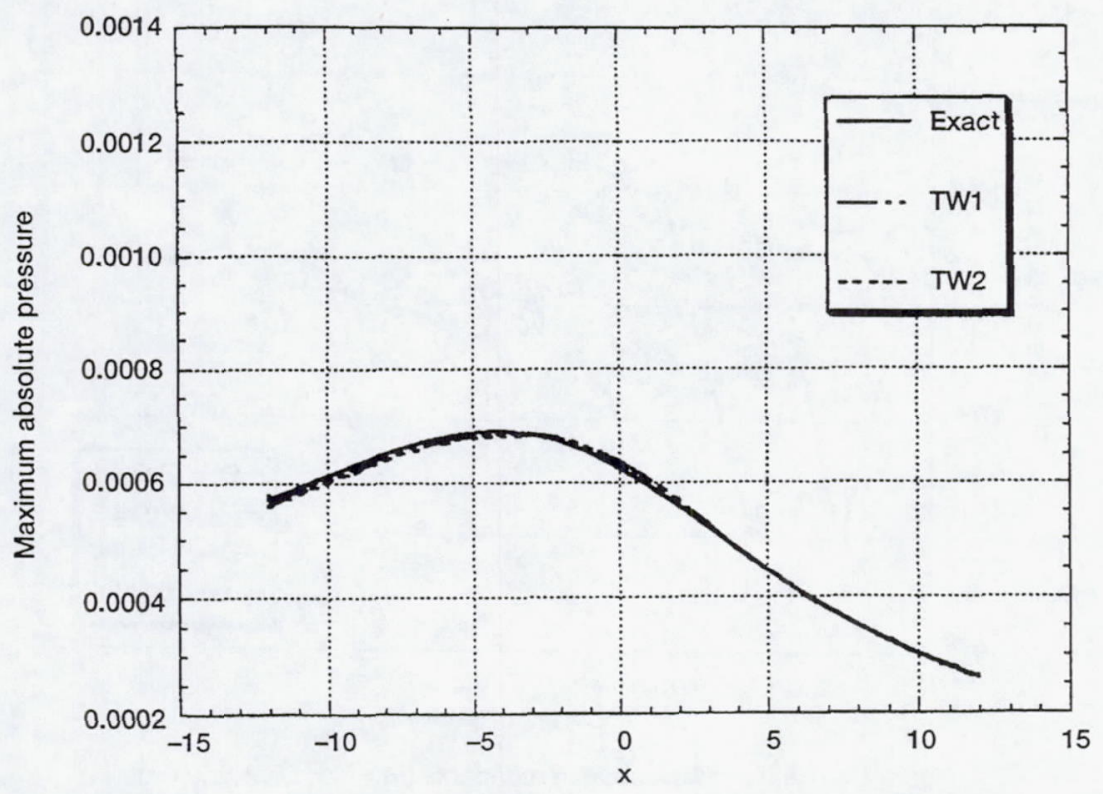

Figure 20.-Uniform flow about a monopole ( $\mathrm{M}=0.5$; Tam and Webb). 


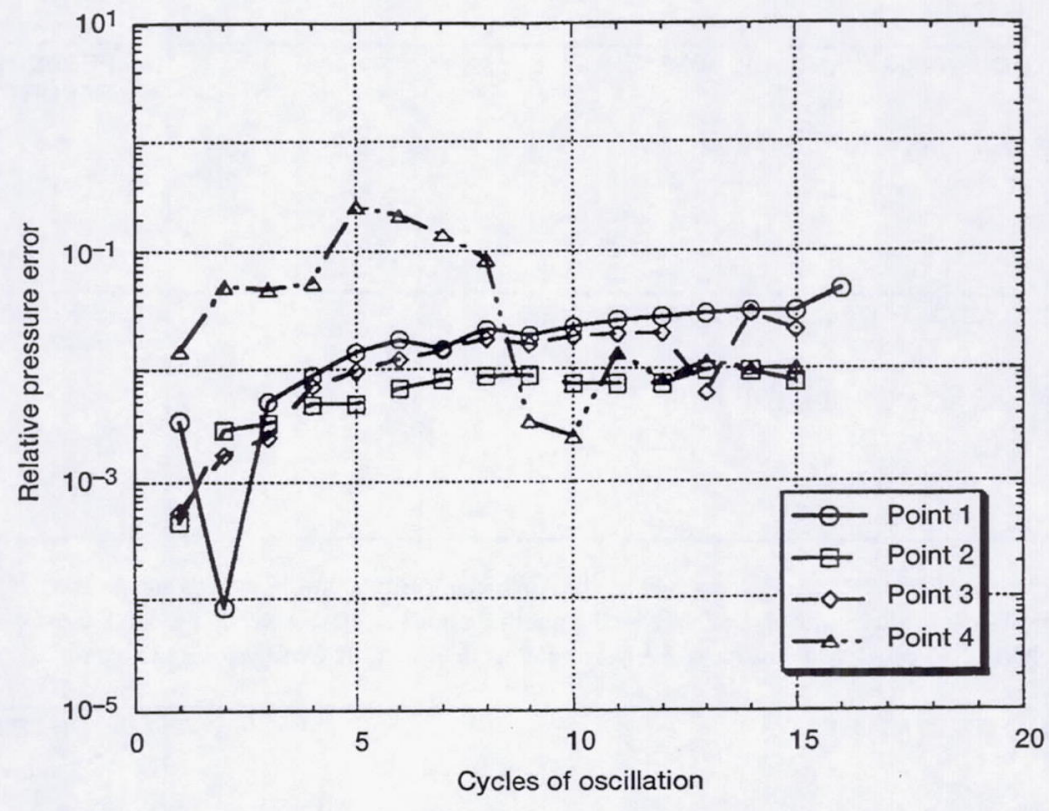

Figure 21.-Uniform flow about a monopole ( $\mathrm{M}=0.5$; Tam 2 nd order). 
Public reporting burden for this collection of information is estimated to average 1 hour per response, including the time for reviewing instructions, searching existing data sources, gathering and maintaining the data needed, and completing and reviewing the collection of information. Send comments regarding this burden estimate or any other aspect of this collection of information, including suggestions for reducing this burden, to Washington Headquarters Services, Directorate for Information Operations and Reports, 1215 Jeffer
Davis Highway, Suite 1204, Arlington, VA 22202-4302, and to the Office of Management and Budget, Paperwork Reduction Project (0704-0188), Washington, DC 20503.

\begin{tabular}{|l|l|l|}
\hline 1. AGENCY USE ONLY (Leave blank) & $\begin{array}{r}\text { 2. REPORT DATE } \\
\text { December } 1994\end{array}$ & $\begin{array}{r}\text { 3. REPORT TYPE AND DATES COVERED } \\
\text { Technical Memorandum }\end{array}$ \\
\hline
\end{tabular}

\section{TITLE AND SUBTITLE}

5. FUNDING NUMBERS

Evaluation of Boundary Conditions for Computational Aeroacoustics

6. AUTHOR(S)

WU-505-90-5K

R. Hixon, S.-H. Shih, and R.R. Mankbadi

7. PERFORMING ORGANIZATION NAME(S) AND ADDRESS(ES)

National Aeronautics and Space Administration

Lewis Research Center

Cleveland, Ohio 44135-3191

8. PERFORMING ORGANIZATION REPORT NUMBER

E-8950

9. SPONSORING/MONITORING AGENCY NAME(S) AND ADDRESS(ES)

National Aeronautics and Space Administration

Washington, D.C. 20546-0001

10. SPONSORING/MONITORING AGENCY REPORT NUMBER

NASA TM-106645

ICOMP-94-11

AIAA-95-0160

11. SUPPLEMENTARY NOTES

Prepared for the 33rd Aerospace Sciences Meeting sponsored by the American Institute of Aeronautics and Astronautics, Reno, Nevada, January 5-12, 1995. R. Hixon and S.-H. Shih, Institute for Computational Mechanics in Propulsion, NASA Lewis Research Center (work funded under NASA Cooperative Agreement NCC3-233); R.R. Mankbadi, NASA Lewis Research Center. ICOMP Program Director, Louis A. Povinelli, organization code $2600,(216)$ 433-5818.

12a. DISTRIBUTION/AVAILABILITY STATEMENT

12b. DISTRIBUTION CODE

Unclassified - Unlimited

Subject Category 34

\section{ABSTRACT (Maximum 200 words)}

The performance of three acoustic boundary condition formulations are investigated. The effect of implementation differences are also studied. Details of all implementations are given. Results are shown for the acoustic field of a monopole in a uniform freestream.

\section{SUBJECT TERMS}

Boundary conditions; Computational fluid dynamics; Computational aero-acoustics;

Acoustics; Jet noise

15. NUMBER OF PAGES

31

16. PRICE CODE

SECURITY CLASSIFICATION

OF REPORT

18. SECURITY CLASSIFICATION OF THIS PAGE

Unclassified

Unclassified

19. SECURITY CLASSIFICATION OF ABSTRACT

$\mathrm{A} 03$

Unclassified

20. LIMITATION OF ABSTRACT 